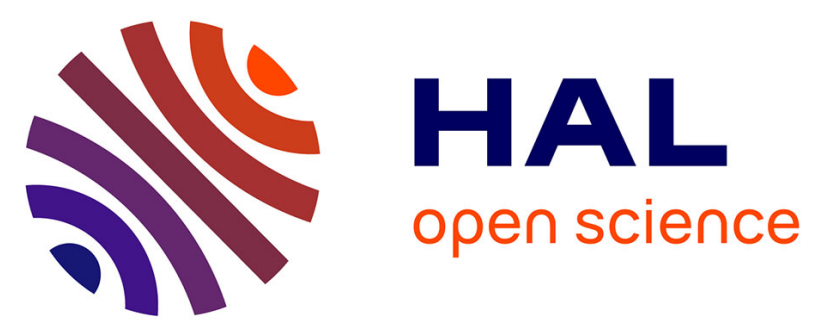

\title{
Eulerian and Lagrangian Ice Crystal Trajectory Simulations in a Generic Turbofan Compressor
}

Ellen Norde, Jean-Mathieu Senoner, Edwin Theodorus Antonius van Der Weide, Pierre Trontin, Hendrik Willem Marie Hoeijmakers, Philippe Villedieu

\section{- To cite this version:}

Ellen Norde, Jean-Mathieu Senoner, Edwin Theodorus Antonius van Der Weide, Pierre Trontin, Hendrik Willem Marie Hoeijmakers, et al.. Eulerian and Lagrangian Ice Crystal Trajectory Simulations in a Generic Turbofan Compressor. Journal of Propulsion and Power, 2018, pp.1-15. 10.2514/1.B36916 . hal-02055824

\section{HAL Id: hal-02055824 \\ https://hal.science/hal-02055824}

Submitted on 4 Mar 2019

HAL is a multi-disciplinary open access archive for the deposit and dissemination of scientific research documents, whether they are published or not. The documents may come from teaching and research institutions in France or abroad, or from public or private research centers.
L'archive ouverte pluridisciplinaire HAL, est destinée au dépôt et à la diffusion de documents scientifiques de niveau recherche, publiés ou non, émanant des établissements d'enseignement et de recherche français ou étrangers, des laboratoires publics ou privés. 


\title{
Eulerian and Lagrangian Ice Crystal Trajectory
}

\section{Simulations in a Generic Turbofan Compressor}

\author{
E. Norde ${ }^{\mathrm{a}}$ \\ ADSE Consulting and Engineering, 2132 LR Hoofddorp, the Netherlands \\ J.-M. Senoner ${ }^{\mathrm{b}}$ \\ ONERA / DMPE - Université de Toulouse, F-31055 Toulouse, France \\ E.T.A. van der Weide ${ }^{\mathrm{c}}$ \\ University of Twente, 7522 NB Enschede, the Netherlands \\ P. Trontin ${ }^{\mathrm{d}}$ \\ ONERA / DMPE - Université de Toulouse, F-31055 Toulouse, France \\ H.W.M. Hoeijmakers ${ }^{\mathrm{e}}$ \\ University of Twente, 7522 NB Enschede, the Netherlands \\ P. Villedieu ${ }^{\mathrm{f}}$ \\ ONERA / DMPE - Université de Toulouse, F-31055 Toulouse, France
}

This study provides a comparison between an Eulerian and a Lagrangian approach for simulation of ice crystal trajectories and impact in a generic turbofan compressor. The engine-like geometry consists of a one-and-a-half stage (stator-rotor-stator) compressor in which the computed air flow is steady and inviscid. Both methods apply the same models to evaluate ice crystal dynamics, mass and heat transfer, and phase change along ice crystal trajectories. The impingement of the crystals on the blade surfaces is modeled assuming full deposition for comparison and validation purposes. Moreover, the effect of ice crystal diameter and sphericity variations on impinging mass flux and

\footnotetext{
a Technical Specialist, ellen.norde@adse.eu (research carried out as PhD student, Engineering Fluid Dynamics Group, University of Twente).

b Research Engineer, Multiphysics and Energetics Department, jean-mathieu.senoner@onera.fr.

c Assistant Professor, Engineering Fluid Dynamics Group, AIAA Senior Member.

d Research Engineer, Multiphysics and Energetics Department

e Professor, Engineering Fluid Dynamics, AIAA Senior Member.

${ }^{\mathrm{f}}$ Scientific Director, Multiphysics and Energetics Department
} 
particle melting ratio is briefly assessed. Then, a more realistic wall interaction model predicts rebound, shattering or deposition as a function of impact parameters that is applied. When the full deposition model is activated, excellent agreement is observed between Eulerian and Lagrangian approaches for the impinging mass flux profiles on each blade while moderate differences appear for the melting curves. However, significant differences appear between both approaches when using the more realistic wall interaction model. The analysis of these results highlights classic limitations of standard Eulerian and Lagrangian methods for this type of applications.

\section{Nomenclature}

Roman symbols

$$
\begin{aligned}
& A=\text { surface area }\left[\mathrm{m}^{2}\right] \\
& L \quad=\text { latent heat }\left[\mathrm{J} \mathrm{kg}^{-1}\right] \\
& B_{M}=\text { Spalding mass number } \\
& \mathcal{L}=\text { dimensionless impact parameter } \\
& B_{T} \quad=\text { Spalding thermal number } \\
& \text { LWC }=\text { Liquid Water Content }\left[\mathrm{g} \mathrm{m}^{-3}\right] \\
& C_{D}=\text { drag coefficient } \\
& l_{s}=\text { curvilinear length scale }[\mathrm{m}] \\
& c \quad=\operatorname{chord}[\mathrm{m}] \\
& M \quad=\text { Mach number } \\
& c_{p, x}=\text { specific heat capacity of species } x\left[\mathrm{~J} \mathrm{~kg}^{-1} \mathrm{~K}^{-1}\right] m \quad=\text { particle mass }[\mathrm{kg}] \\
& c_{p} \quad=\text { pressure coefficient } \\
& \dot{m}=\text { mass rate }\left[\mathrm{kg} \mathrm{s}^{-1}\right] \\
& D_{v} \quad=\text { vapor diffusivity in air }\left[\mathrm{m}^{2} \mathrm{~s}^{-1}\right] \\
& N \quad=\text { number of (particles, bins,...) } \\
& d_{p} \quad=\text { particle equivalent diameter }[\mathrm{m}] \\
& n \quad=\text { number density }\left[\mathrm{m}^{-3}\right] \\
& e_{\sigma} \quad=\text { surface energy }\left[\mathrm{J} \mathrm{m}^{-2}\right] \\
& \vec{n} \quad=\text { normal unit vector } \\
& \vec{f}_{D}=\operatorname{drag} \text { force }[\mathrm{N}] \\
& \mathrm{Nu} \quad=\text { Nusselt number } \\
& \overrightarrow{f_{I}}=\text { inertial force }[\mathrm{N}] \\
& p \quad=\text { pressure }[\mathrm{Pa}] \\
& h \quad=\operatorname{spacing}[\mathrm{m}] \\
& \operatorname{Pr} \quad=\text { Prandtl number } \\
& \text { IWC }=\text { Ice Water Content }\left[\mathrm{g} \mathrm{m}^{-3}\right] \\
& \dot{Q}_{c}=\text { conductive heat transfer rate }\left[\mathrm{J} \mathrm{s}^{-1}\right] \\
& K \quad=\text { experimentally fitted parameter } \\
& Q_{s} \quad=\text { activation energy }\left[\mathrm{J} \mathrm{mol}^{-1}\right] \\
& k=\text { heat conductivity }\left[\mathrm{W} \mathrm{K}^{-1}\right] \\
& R=\text { universal gas constant }\left[\mathrm{J} \mathrm{K}^{-1} \mathrm{~mol}^{-1}\right]
\end{aligned}
$$




$$
\begin{aligned}
& \mathcal{R}_{d}=\text { random number between } 0 \text { and } 1 \quad T \quad=\text { temperature }[\mathrm{K}] \\
& \mathcal{R}_{\phi}=\text { random number between } 0.7 \text { and } 0.9 \quad t \quad=\text { time }[\mathrm{s}] \\
& \text { Re }=\text { Reynolds number } \quad \text { TWC }=\text { Total Water Content }\left[\mathrm{g} \mathrm{m}^{-3}\right] \\
& \mathrm{RH}=\text { Relative Humidity }[\%] \quad \vec{t} \quad=\text { tangential unit vector } \\
& r=\operatorname{radius}[\mathrm{m}] \quad \vec{v} \quad=\text { velocity }\left[\mathrm{m} \mathrm{s}^{-1}\right] \\
& \mathrm{Sc}=\text { Schmidt number } \quad w \quad=\text { weight factor } \\
& \mathrm{Sh}=\text { Sherwood number } \quad(x, y, z)=\text { Cartesian coordinates }[\mathrm{m}] \\
& s \quad=\text { curvilinear coordinate }[\mathrm{m}] \quad y \quad=\text { mass fraction }
\end{aligned}
$$

Greek symbols

$$
\begin{aligned}
& \alpha=\text { particle volume fraction } \\
& \dot{\alpha}=\text { particle volume fraction rate }\left[\mathrm{s}^{-1}\right] \\
& \beta=\text { catching efficiency } \\
& \varepsilon=\text { sticking efficiency } \\
& \epsilon=\text { characteristic length scale }[\mathrm{m}] \\
& \eta=\text { melting ratio }
\end{aligned}
$$

\begin{tabular}{|c|c|c|c|c|}
\hline$a$ & $=$ air & $m$ & $=$ melting & tot $=$ total \\
\hline bin & $=$ particle bin & $\max$ & $=$ maximum value & $u p=$ upper value \\
\hline$f$ & $=$ fusion & $n$ & $=$ normal & $v=$ vapor/vaporization \\
\hline$i$ & $=\mathrm{ice}$ & $p$ & $=$ particle & $w=$ water \\
\hline$i c$ & $=$ ice crystal & $s$ & $=$ secondary & $0=$ initial value \\
\hline$i m p$ & $=$ impinging & sub & $=$ sublimation & $=$ re-emitted particle \\
\hline low & $=$ lower value & $t$ & $=$ tangential & $\infty=$ free stream \\
\hline
\end{tabular}$$
\mu=\text { dynamic viscosity }\left[\mathrm{kg} \mathrm{m}^{-1} \mathrm{~s}^{-1}\right]
$$$$
\xi \quad=\text { restitution coefficient }
$$$$
\rho=\text { density }\left[\mathrm{kg} \mathrm{m}^{-3}\right]
$$$$
\tau=\text { characteristic time scale }[\mathrm{s}]
$$$$
\Phi=\text { particle sphericity }
$$$$
\Phi_{\perp}=\text { particle crosswise sphericity }
$$

\section{Subscripts/Superscripts}

\section{Introduction}

In the last decade a growing number of research efforts have been undertaken to understand jet engine power loss and damage caused by icing due to ingestion of ice crystals. The first reported 
icing events date from 1990 and with on average seven incidents occurring each year [1], engine icing remains a serious threat to aviation safety. The majority of these events occurred near convective clouds with high concentrations of ice crystals at a median altitude of 10.7 kilometers. The presence of small ice crystals is not registered by radar or ice detector and airframe icing is not observed by the flight crew since ice crystals bounce off cold surfaces. However, near warmer engine parts and heated probes, ice crystals will melt partially and stick to the impinged surface. This may result in engine stall, flameout or even engine damage. Contrary to aircraft icing caused by supercooled (large) droplets for which advanced prediction tools such as LEWICE [2], FENSAP-ICE [3, 4] and ONICE $[5,6]$ are currently available, ice accretion due to ice crystals is not yet fully understood. Therefore, further research efforts need to be undertaken, amongst others through the development of dedicated numerical tools.

The research in this paper is carried out in the framework of European project HAIC [7], acronym for High Altitude Ice Crystals. The focus of the present study is on the comparison of Lagrangian and Eulerian approaches for the calculation of ice crystal trajectories in an engine-like geometry. Other comparisons of results of Lagrangian and Eulerian approaches were published for, amongst others, an aeronautical combustion chamber [8], a pitching airfoil [9] and a particle separator [10]. However, to the authors' knowledge, such a comparison was never performed to analyse ice crystal dynamics in a generic turbofan compressor. The purpose of this comparison is threefold. First, details on the implementation of the models related to the evolution of the ice crystals are provided and compared for both approaches. Second, the comparison of the results obtained with both approaches serves code validation purposes. Finally, advantages and limitations of each approach with respect to the present application may be highlighted. The last point is particularly relevant as Lagrangian and Eulerian approaches display complementary advantages which make the choice of either not obvious.

The Lagrangian approach is based on a direct resolution of the Boltzmann-type equation describing the evolution of the spray density function [11]. The spray is described using a statistical sample of particles. The spray density function is then approximated as a sum of Dirac delta functions centered at each sampled particle. This approach allows for a straightforward implementation 
of physical models since properties are available at the particle scale. Furthermore, polydispersity is naturally handled within the Lagrangian approach. However, its accuracy is directly dependent on the number of sampled particles and convergence will be slow in the general case, i.e. of the order $N_{p}^{-1 / 2}$ according to the central limit theorem, with $N_{p}$ the number of sampled particles. When coupling a Lagrangian approach for the dispersed phase to an Eulerian approach for the carrier phase, interpolations from the mesh points to the particle positions and vice versa are necessary since both do not necessarily coincide. Moreover, local perturbations of the carrier phase induced by the particles ("two-way coupling") may lead to stiff source terms which cannot easily be handled through implicit numerical techniques. Finally, achieving efficient parallelization is problematic in a Lagrangian framework. When the flow field induces spatial particle accumulations, the computational load associated with each processor will be unevenly balanced if the same domain decomposition is applied to both the Eulerian mesh used for the carrier phase and for the particles.

The Eulerian method solves balance equations for a given set of moments of the spray density function [12]. The derivation of the Eulerian balance equations involves averages in phase space and generally requires additional spatial or ensemble averages. The Eulerian approach is generally more efficient in terms of computational expense. In particular, its cost does not depend on the number of particles present in the target configuration. Furthermore, ensuring an even computational load balancing between processors in parallel computations is straightforward as the same domain decomposition may be applied for the resolution of carrier and dispersed phase. On the other hand, the accurate treatment of complex physical phenomena such as the crossing of two jets laden with particles or the interaction between particles and walls is more tedious. Accounting for such phenomena in an Eulerian framework requires the ability to locally, i.e. per control volume, define more than one particle velocity [13]. Furthermore, modeling polydispersity is not as straightforward as for the Lagrangian approach. In order to account for different particle sizes locally, the diameter space may either be discretized into bins or into sections. The Eulerian system of equations for the dispersed phase then needs to be solved for each bin or section, somewhat mitigating the computational advantage inherent to the Eulerian approach.

The Euler-Lagrange simulations performed in the present work rely on ONERA's multiphysics 
solver CEDRE [14]. Further numerical details on CEDRE's Lagrangian particle solver may be found in [15]. The Eulerian ice crystal simulations are performed with University of Twente's in-house code MooseMBIce. Building on the experience with numerical simulation of supercooled large droplets [16], the capabilities of MooseMBIce were recently extended for ice crystals and the method was coupled to the in-house Navier-Stokes solver. Further numerical details on the present Euler-Euler simulations may be found in [17].

The present article is organized as follows. First, the equations governing particle dynamics, heat and mass transfer, as well as phase change along trajectories are provided. Then, the target geometry and the numerical results obtained with both approaches are compared and discussed. The main findings and outlooks are summarized in the conclusion.

\section{Ice crystal trajectories}

Icing due to ice crystals is different from icing due to supercooled droplets in that the effects of phase change and particle shape on the trajectory have to be considered. Ice crystals are subject to a warm environment. Therefore, phase change (melting/evaporation/sublimation) and mass/ heat exchange phenomena with the surrounding air need to be taken into account. Furthermore, ice crystals encountered in the vicinity of convective clouds display shapes that are far from spherical. The expressions governing the motion, mass transfer, heat transfer and phase change need to be modified such that the effect of their shape is explicitly taken into account. These extensions were described previously for both Lagrangian $[18,19]$ and Eulerian methods [20-23]. In this section the trajectory models are briefly described to underline the specifics of each approach.

\section{A. Particle motion}

The particle motion is influenced by several forces such as drag, gravity, buoyancy, virtual mass, Basset history force and lift [24]. Due to the high ratio of particle density and air density and the small particle sizes found in the present applications, only drag forces need to be considered, in a fixed frame of reference, as the magnitude of the other forces is negligible in comparison. In order to account for the presence of moving parts in the target geometry, the particle equations of motion are solved in a rotating frame of reference while maintaining the geometry fixed. Such description yields 
additional volumetric forces, namely Coriolis and centrifugal forces. The conversion from fixed to rotating frames of reference is then handled via mixing planes. The corresponding equations for Lagrangian motion and Eulerian momentum are given in Table 2 and 3, respectively. In these tables the terms $\vec{f}_{D}$ and $\vec{f}_{I}$ correspond to the drag forces and inertial forces, respectively. The inertial forces $\vec{f}_{I}$ are due to the motion of the reference frame.

A number of relations for the drag coefficient $C_{D}$ can be found in [25-27]. These relations take the particle shape into account by means of a particle sphericity or aspect ratio. Benchmark test cases performed within the HAIC project showed that these drag relations yielded very similar results. In the present work, the drag coefficient proposed by Ganser [26] is chosen because of its relative simplicity, while including two sphericity parameters: the particle sphericity $\Phi$ and the crosswise particle sphericity $\Phi_{\perp}$. The particle sphericity $\Phi$ is the ratio of the surface area of a sphere with the same volume as the particle and the true surface area $A$ of that particle, i.e. $\Phi=\pi d_{p}^{2} / A$. The crosswise sphericity $\phi_{\perp}$ is the ratio of the cross-sectional area of the volume-equivalent sphere and the area of the projection of the considered particle on a plane perpendicular to the flow. Ganser [26] proposed a drag relation that depends on a Stokes shape factor $K_{1}$ and a Newton shape factor $K_{2}$, see Eq. (1). These shape factors are fitted to experimental data and are a function of the particle sphericity and of the projected area in the direction of motion, see Table 1 . The second part of shape factor $K_{1}$ is non-zero if the particle is settling in a tube with diameter $d_{\text {tube }}$ and is to be omitted when the tube is absent.

$$
C_{D}=\frac{24}{\operatorname{Re} K_{1}}\left(1+0.1118\left(\operatorname{Re} K_{1} K_{2}\right)^{0.6567}\right)+\frac{0.4305 K_{2}}{1+\frac{3305}{\operatorname{Re} K_{1} K_{2}}}, \quad \text { for } \quad \operatorname{Re} K_{1} K_{2} \leq 10^{5} .
$$

The particle Reynolds number is given by:

$$
\operatorname{Re}=\frac{\rho_{a} d_{p}\left|\vec{v}_{a}-\vec{v}_{p}\right|}{\mu_{a}}
$$

where $\rho_{a}$ is the density of air, $d_{p}$ is the particle volume-equivalent diameter, $\mu_{a}$ is the dynamic viscosity of air, and $\vec{v}_{a}$ and $\vec{v}_{p}$ are the air and particle velocity, respectively.

\section{B. Particle heat transfer and phase change}

In the present simulations, along the particle trajectory three phase change processes may occur: sublimation, melting and evaporation. These three phase changes are driven by diffusive mass and 
Table 1: Stokes' and Newton's shape factors [26].

\begin{tabular}{lll}
\hline \hline Shape & $K_{1}$ & $K_{2}$ \\
\hline Isometric & $K_{1}=\left(\frac{1}{3}+\frac{2}{3} \Phi^{-1 / 2}\right)^{-1}-2.25 \frac{d_{p}}{d_{\text {tube }}}$ & $K_{2}=10^{1.8148\left(-\log _{10} \Phi\right)^{0.5743}}$ \\
Nonisometric & $K_{1}=\left(\frac{1}{3} \Phi_{\perp}^{-1 / 2}+\frac{2}{3} \Phi^{-1 / 2}\right)^{-1}-2.25 \frac{d_{p}}{d_{\text {tube }}}$ & $K_{2}=10^{1.8148\left(-\log _{10} \Phi\right)^{0.5743}}$ \\
\hline \hline
\end{tabular}

heat transfer phenomena between the particles and the surrounding gas.

The governing equations are described in the following, with a particular focus on differences between the Euler-Lagrange method and the Euler-Euler method. The Lagrangian solver implements particle sublimation/evaporation using Spalding's standard model [28]. The mass flux of water vapor at the particle's surface due to sublimation/evaporation reads

$$
\frac{d m_{p}}{d t}=-\pi d_{p} \operatorname{Sh} \rho_{a} D_{v} \ln \left(1+B_{M}\right)
$$

with $d_{p}$ the particle diameter. The expressions for the Sherwood number Sh and the diffusivity $D_{v}$ of the vapor species $v$ are provided further in Eqs. (15) and (10), respectively. When considering the evaporation of a single species, $B_{M}$ simplifies to:

$$
B_{M}=\frac{y_{v, s}-y_{v, a}}{1-y_{v, a}}
$$

where $y_{v, s}$ and $y_{v, a}$ denote water vapor mass fractions at the particle surface and at the outside of the water vapor layer surrounding the particle (infinity for a fluid at rest), respectively. In icing applications, vapor mass fractions generally remain small (of the order of $10^{-3}$ ) due to the low temperature of the carrier phase and low particle volume fractions (of the order of $10^{-6}$ ). Thus, $y_{v, s} \ll 1$ and $y_{v, a} \ll 1$. It follows that $B_{M} \ll 1,1-y_{v, a} \approx 1$ and finally

$$
\ln \left(1+B_{M}\right) \approx B_{M} \approx\left(y_{v, s}-y_{v, a}\right)
$$

Equation (3) may then be approximated as:

$$
\frac{d m_{p}}{d t}=\pi d_{p} \operatorname{Sh} \rho_{a} D_{v}\left(y_{v, s}-y_{v, a}\right)
$$

Because CEDRE is also used for aeronautic combustion and solid propulsion applications for which the aforementioned simplifications are not applicable [29], the Lagrangian method uses Eq. (3), 
while the Eulerian method relies on the simplified formulation of Eq. (6). It is verified numerically that both equations lead to nominally identical results for the occurring vapor mass fraction ranges. The model for the melting of the ice particles is based on work of Mason [30] on the melting of hailstones. The energy equation governing the transfer of heat between the air and the particle during melting can be written as:

$$
\dot{Q}_{c}=\dot{m}_{v} L_{v}+\dot{m}_{f} L_{f} \Longrightarrow \pi d_{p} \mathrm{Nu} k_{a} \frac{\ln \left(1+B_{T}\right)}{B_{T}}\left(T_{a}-T_{f}\right)=\dot{m}_{v} L_{v}\left(T_{f}\right)+\dot{m}_{f} L_{f}\left(T_{f}\right)
$$

with $B_{T}$ defined as:

$$
B_{T}=\frac{\left(T_{a}-T_{f}\right) c_{p, v}}{L_{v}\left(T_{f}\right)}
$$

Here, $\dot{m}_{f}$ is the fusion mass flow rate, $\dot{m}_{v}$ is the evaporating mass flow rate and $L_{f}$ and $L_{v}$ are the latent heats of fusion and evaporation, respectively. In Eq. (7) the kinetic energy associated with the velocity of the particle surface is neglected because it is typically very small compared to the enthalpy $L_{f}$ associated with the phase change of the particle. The heat conduction is governed by the particle equivalent diameter $d_{p}$, the heat conduction coefficient of air $k_{a}$, the particle Nusselt number $\mathrm{Nu}$ and the difference between the air temperature $T_{a}$ and the fusion temperature $T_{f}$. In Eq. (8) $c_{p, v}$ denotes the heat capacity of the gaseous vapor species at constant pressure. The conductive heating term $\dot{Q}_{c}$ may be simplified analogously to the mass transfer term, assuming $B_{T} \ll 1$, yielding:

$$
\dot{Q}_{c}=\dot{m}_{v} L_{v}+\dot{m}_{f} L_{f} \Longrightarrow \pi d_{p} \mathrm{Nu} k_{a}\left(T_{a}-T_{f}\right)=\dot{m}_{v} L_{v}\left(T_{f}\right)+\dot{m}_{f} L_{f}\left(T_{f}\right) .
$$

Consistently, the Euler-Lagrange simulations solve Eq. (7), while the Euler-Euler simulations rely on the simplified description of Eq. (9).

As is apparent from Eq. (3) and Eq. (9), the evaluation of melting and evaporation rates requires the knowledge of transport properties at the particle location, in particular the vapor diffusivity $D_{v}$ and heat conduction $k_{a}$ of the vapor-air mixture. In the Euler-Lagrange simulations, the vapor diffusivity $D_{v}$ is obtained via an imposed Schmidt number for the vapor species:

$$
D_{v}=\frac{\overline{\mu_{v}}}{\rho_{a} \mathrm{Sc}}
$$


where the vapor viscosity $\overline{\mu_{v}}$ is obtained from Sutherland's law for vapor. On the other hand, the Euler-Euler simulations rely on Schirmer's law [31]. Evidence suggests that the slight numerical differences (of the order of a few percent) induced by the use of these different formulae explain the differences observed for the melting ratios obtained by the two approaches. Deploying a unified set of thermophysical models for the carrier phase of both approaches, however, was beyond the scope of the present comparison.

Properties of the vapor-air mixture are based on a mass-weighted average in the Euler-Lagrange simulations. In particular, the heat conductivity is evaluated as:

$$
\overline{k_{a}}=\sum_{i} y_{i} k_{a, i}
$$

while the Euler-Euler simulations use just air properties. Given the very low vapor mass fractions encountered in the present simulations, the resulting differences are again negligible.

During melting the particle will evolve towards a spherical shape as surface tension effects come into play. This complex process is not sufficiently understood yet. Therefore, a simple model $[18,19]$ is applied to approximate this evolution. In this approximation the sphericity is interpolated between $\Phi_{0}$, its original value, and 1 , the sphericity of a perfectly spherical particle.

$$
\begin{gathered}
\text { Lagrangian: } \Phi=\left(\frac{m_{p, i}}{m_{p}}\right) \Phi_{0}+\left[1-\left(\frac{m_{p, i}}{m_{p}}\right)\right] \\
\text { Eulerian: } \Phi=\left(\frac{\rho_{i} \alpha_{i}}{\rho_{p} \alpha_{p}}\right) \Phi_{0}+\left[1-\left(\frac{\rho_{i} \alpha_{i}}{\rho_{p} \alpha_{p}}\right)\right]
\end{gathered}
$$

where

$$
m_{p}=\pi \rho_{p} \frac{d_{p}^{3}}{6}, \quad m_{p, i}=\pi \rho_{i} \frac{d_{p, i}^{3}}{6}, \quad \alpha_{p}=\alpha_{i}+\alpha_{w}
$$

The corresponding equations for particle mass and energy for each of the three stages are given in Table 2 for the Lagrangian approach and in Table 3 for the Eulerian approach.

For the Nusselt number a relation that was derived in [32] is applied, i.e.

$$
\mathrm{Nu}=2 \sqrt{\Phi}+0.55 \operatorname{Pr}^{1 / 3} \Phi^{1 / 4} \sqrt{\operatorname{Re}}
$$

This equation can be seen as an extension to non-spherical particles of the Frössling relation [33] for spherical particles. The Sherwood number Sh of the vapor species is linked to the Schmidt number 
Table 2: Lagrangian trajectory model

\begin{tabular}{|c|c|c|c|c|}
\hline & Motion & $m_{p} \frac{d \vec{v}_{p}}{d t}$ & $=$ & $\vec{f}_{D}+\vec{f}_{I}$ \\
\hline \multirow[t]{2}{*}{$T_{p}<T_{f}$} & Mass & $\frac{d m_{p}}{d t}$ & $=$ & $-\pi d_{p} \frac{\mathrm{Sh}}{\Phi} \rho_{a} D_{v} \ln \left(1+B_{M}\right)=-\dot{m}_{s u b}$ \\
\hline & Temperature & $m_{p} c_{p, i} \frac{d T_{p}}{d t}$ & $=$ & $\pi d_{p} \frac{\mathrm{Nu}}{\Phi} k_{a} \frac{\ln \left(1+B_{T}\right)}{B_{T}}\left(T_{a}-T_{p}\right)-\dot{m}_{s u b}\left(L_{f}+L_{v}\right)$ \\
\hline \multirow[t]{2}{*}{$T_{p}=T_{f}$} & Mass: particle & $\frac{d m_{p}}{d t}$ & $=$ & $-\pi d_{p} \frac{\mathrm{Sh}}{\Phi} \rho_{a} D_{v} \ln \left(1+B_{M}\right)=-\dot{m}_{v}$ \\
\hline & Mass: ice core & $L_{f} \frac{d m_{p, i}}{d t}$ & $=$ & $-\dot{m}_{f} L_{f}=-\pi d_{p} \frac{\mathrm{Nu}}{\Phi} k_{a} \frac{\ln \left(1+B_{T}\right)}{B_{T}}\left(T_{a}-T_{f}\right)+\dot{m}_{v} L_{v}$ \\
\hline \multirow[t]{2}{*}{$T_{p}>T_{f}$} & Mass & $\frac{d m_{p}}{d t}$ & $=$ & $-\pi d_{p} \operatorname{Sh} \rho_{a} D_{v} \ln \left(1+B_{M}\right) \quad=\quad-\dot{m}_{v}$ \\
\hline & Temperature & $m_{p} c_{p, w} \frac{d T_{p}}{d t}$ & $=$ & $\pi d_{p} \mathrm{Nu} k_{a} \frac{\ln \left(1+B_{T}\right)}{B_{T}}\left(T_{a}-T_{p}\right)-\dot{m}_{v} L_{v}$ \\
\hline
\end{tabular}

Table 3: Eulerian trajectory model

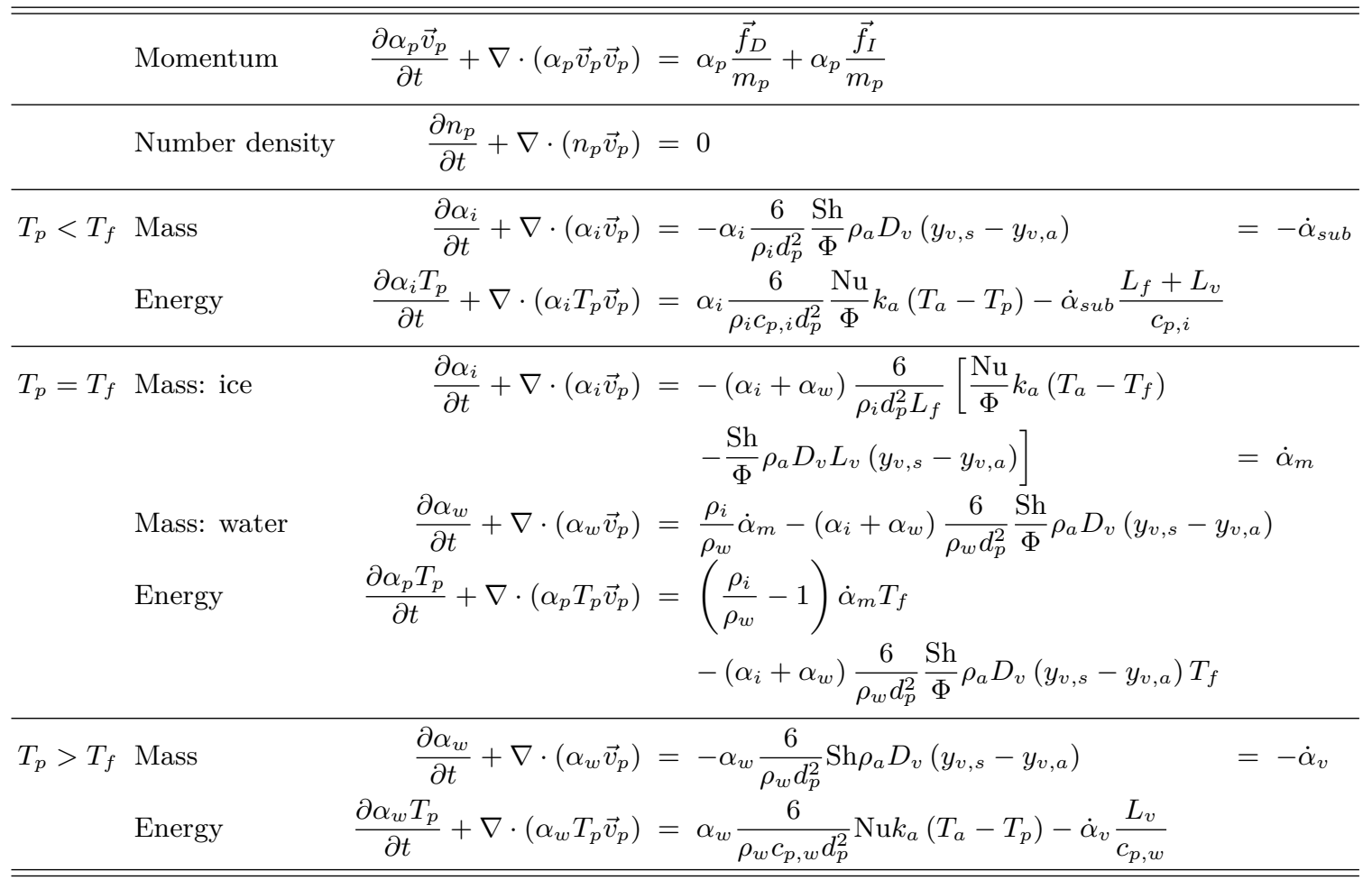

Sc and Nusselt number Nu by the Chilton-Colburn analogy:

$$
\mathrm{Sh}=2 \sqrt{\Phi}+0.55 \mathrm{Sc}^{1 / 3} \Phi^{1 / 4} \sqrt{\operatorname{Re}}
$$

The results from the model for the phase change described in this section are compared to the results from experiments performed by Hauk [32, 34]. The agreement between the measured and 
computed melting times and final droplet diameter was very good [32].

To summarize, the Lagrangian trajectory approach solves four trajectory equations for particle mass $m_{p}$, ice core mass $m_{p, i}$, velocity $\vec{v}_{p}$ and temperature $T_{p}$. In the Eulerian approach five equations are solved: ice volume fraction $\alpha_{i}$, water volume fraction $\alpha_{w}$, particle number density $n_{p}$, particle velocity $\vec{v}_{p}$ and particle temperature $T_{p}$.

\section{Ice crystal impingement}

The interaction of a (partially melted) ice crystal with a wall is a complex process. The impact event will result in different outcomes depending on the surface properties (dry or wet wall, surface roughness, surface temperature) as well as the state of the ice crystal (degree of melting, velocity). The model for ice crystal impact applied in the present work was described previously in [18, 19]. The impact process depends on two parameters, a dimensionless parameter related to the kinetic energy that defines the different impact regimes, and a probability of particle deposition. The impact model and the secondary particle characteristics are described in sections III A and IIIB, respectively. Furthermore, in the Eulerian approach the treatment of the secondary particle reinjection is not straightforward. The implementation of the secondary cloud in the Lagrangian and Eulerian method is treated in sections III C and III D, respectively.

\section{A. Impact model}

An ice crystal that impacts a surface can either stick to the surface, rebound from the surface or shatter into a number of smaller fragments. Particle impact on a dry surface was investigated experimentally for hailstones [35-37] and for ice crystals [34, 38, 39]. Vidaurre and Hallett [39] experimentally investigated the breakup of 5-320 $\mu \mathrm{m}$ sized ice crystals and Guégan et al. [35] investigated the breakup of hailstones with diameters between 12.9 and 42 mm. Both studies showed that the particle kinetic energy based on the normal component of the particle velocity is a good indicator for the impact behavior of the particle. As a result a dimensionless impact parameter or modified Weber number $\mathcal{L}$ is introduced, which is the ratio of kinetic energy and surface energy:

$$
\mathcal{L}=\frac{\rho_{p} d_{p} v_{n}^{2}}{12 e_{\sigma}}
$$


The surface energy $e_{\sigma}$ is defined as

$$
e_{\sigma}(T)=e_{\sigma 0} \exp \left[\frac{Q_{s}}{R}\left(\frac{1}{T_{p}}-\frac{1}{T_{0}}\right)\right]
$$

Vidaurre and Hallett [39] assumed the initial surface energy $e_{\sigma 0}$ to be $0.12 \mathrm{~J} \mathrm{~m}^{-2}$ at $T_{0}=253$ K. Higa et al. [36] investigated the impact of hailstones with radii between 1.4 and $36 \mathrm{~mm}$ and according to the results of their research the activation energy $Q_{s}$ is equal to $48.2 \mathrm{~kJ} / \mathrm{mol}$. An impact model is defined with three possible impact regimes, which depend on the value of parameter $\mathcal{L}$ :

$\mathcal{L}<\mathcal{L}_{1} \quad$ elastic particle bouncing, restitution coefficient $\xi=1$, $\mathcal{L}_{1}<\mathcal{L} \leq \mathcal{L}_{2} \quad$ inelastic particle bouncing, restitution coefficient $\xi<1$, $\mathcal{L}>\mathcal{L}_{2} \quad$ particle fragmentation, restitution coefficient $\xi \ll 1$.

In case of inelastic rebound the particle loses kinetic energy due to crack formation within the particle, but the particle itself remains in one piece. The threshold values $\mathcal{L}_{1}$ and $\mathcal{L}_{2}$ are set to 0.5 and 90, respectively, in accordance with literature data $[35,36,38,39]$.

Upon an impact event the secondary particles can be partly re-emitted into the flow or partly remain on the surface. The presence of liquid water in the air, in the particle, or as a thin film on the wall, will strongly influence the sticking ability of the particles [40, 41]. Experimental observations suggest $[42,43]$ that the particle sticking efficiency mainly depends on the ratio of liquid water content (LWC) and total water content (TWC). The sticking efficiency $\varepsilon$ is defined as the ratio of particle mass that sticks to the surface and the total impinging particle mass. In addition, two separate efficiencies for the mixed-phase regime (ice crystals and droplets) and for the glaciated regime (partially melted ice crystals) [44] need to be considered because it was observed that for the same ratio LWC/TWC the sticking efficiency was higher for melted particles than for mixed-phase conditions. In the present study, only melting crystals are considered so that the evaluation of the sticking efficiency $\varepsilon_{i c}$ reads [19]:

$$
\varepsilon_{i c}=\left(K_{i c}-2\right) \eta^{3}+\left(3-2 K_{i c}\right) \eta^{2}+K_{i c} \eta
$$

where the melting ratio $\eta$ is defined as the ratio between liquid and total water content:

$$
\eta=\frac{\mathrm{LWC}}{\mathrm{TWC}}
$$


The value of the dimensionless parameter $K_{i c}=2.5$ in eq. 18 was adjusted to match experimental data [19].

It should be noted that the present impact model is very basic in that it does not treat the heat exchange at the surface nor any splashing or erosion effects. A heated surface enhances particle melting which has an effect on the particle's sticking ability. The accreted ice layer is influenced by splashing and erosion effects as was shown by experiments [45]. Furthermore, the impact thresholds $\mathcal{L}_{1}$ and $\mathcal{L}_{2}$ are expected to vary with the water amount that is present on the wall or on the particle.

\section{B. Secondary particles}

The secondary particles resulting from a bouncing or shattering event may have properties that are different from those of the parent particle. It is assumed that the particle melting ratio and density remain unchanged during an impact event. The secondary particle diameter, sphericity and velocity, however, are different upon bouncing and shattering of the particles. In case of a bouncing particle, when $\mathcal{L} \leq \mathcal{L}_{2}$, the secondary particle diameter and sphericity remain unchanged. The secondary velocity depends on the normal and on the tangential restitutions coefficient as indicated in the second column in Table 4 . In case of a fragmenting particle, when $\mathcal{L}>\mathcal{L}_{2}$, a new secondary particle diameter and sphericity are introduced. The sphericity is chosen randomly between 0.7 and 0.9 . The secondary diameter depends on an approximation of the diameter $d_{\max }$ of the largest fragment observed in impact experiments [38, 46]:

$$
d_{\text {max }}=\left(\frac{\mathcal{L}_{2}}{\mathcal{L}}\right)^{2 / 11} d_{p}
$$

The velocity of the fragments now depends on the tangential restitution coefficient $\xi_{t}$ and normal restitution coefficients $\xi_{n n}$ and $\xi_{n t}$. The latter two are the fraction of the normal momentum transferred into normal momentum and the fraction of the normal momentum transferred into tangential momentum. The restitution coefficients are derived from results of experiments $[34,38$,

46] and are given in Eq. (21). An overview of secondary particle characteristics in case of shattering is provided in the third column of Table 4. 
Table 4: Characteristics secondary particles $[18,19]$.

\begin{tabular}{lll}
\hline \hline Property & Bounced particle & Fragmented particle \\
\hline Mass $m_{p, s}$ & $(1-\varepsilon) m_{p}$ (Lagrangian) & $(1-\varepsilon) m_{p}$ (Lagrangian) \\
Volume fraction $\alpha_{p, s}$ & $(1-\varepsilon) \alpha_{p} \frac{v_{n}}{v_{n, s}}$ (Eulerian) & $(1-\varepsilon) \alpha_{p} \frac{v_{n}}{v_{n, s}}$ (Eulerian) \\
Diameter $d_{p, s}$ & $d_{p}$ & $\mathcal{R}_{d} d_{m a x}{ }^{*}$ \\
Sphericity $\Phi_{s}$ & $\Phi$ & $\mathcal{R}_{\phi}{ }^{* *}$ \\
Velocity $\vec{v}_{p, s}$ & $\xi_{t}\left[\vec{v}_{p}-\left(\vec{v}_{p} \cdot \vec{n}\right) \vec{n}\right]-\xi_{n n}\left(\vec{v}_{p} \cdot \vec{n}\right) \vec{n}$ & $\xi_{t}\left[\vec{v}_{p}-\left(\vec{v}_{p} \cdot \vec{n}\right) \vec{n}\right]-\left(\vec{v}_{p} \cdot \vec{n}\right)\left(\xi_{n t} \vec{t}+\xi_{n n} \vec{n}\right)$ \\
\hline \hline
\end{tabular}

${ }^{*} \mathcal{R}_{d}$ is a random number between 0 and $1,{ }^{* *} \mathcal{R}_{\phi}$ is a random number between 0.7 and 0.9 .

$$
\xi_{t}=1, \quad \xi_{n t}=0.4\left(1-\sqrt{\frac{\mathcal{L}_{c 2}}{\mathcal{L}}}\right), \quad \xi_{n n}= \begin{cases}1 & \text { if } \mathcal{L} \leq \mathcal{L}_{c 1} \\ \left(\frac{\mathcal{L}_{c 1}}{\mathcal{L}}\right)^{1 / 3} & \text { if } \mathcal{L}>\mathcal{L}_{c 1}\end{cases}
$$

\section{Implementation of Eulerian (post-)impact method}

A standard Eulerian method can handle one particle size, volume fraction, velocity and temperature per grid cell. Therefore, singularities may arise in an Eulerian framework in the event of droplet trajectory crossing [47-49]. The treatment of secondary particles then needs a specific strategy because intersecting flows with different particle properties are introduced by bouncing or shattering. At the surface of impact both an incoming and an outgoing particle may be present. In the present study the incoming (parent) particles and the outgoing (secondary) particles are treated separately. The predefined distribution of parent particles and secondary particles, which can be Gaussian, Rosin-Rammler or bimodal, consists of a discrete set of $N$ points (bins) which do not mutually exchange mass nor momentum. The particles in each individual bin have the same shape, velocity and temperature. A more detailed explanation of approaches using a poly-disperse size distribution is given in [12].

The calculation for the parent particles is carried out first. The trajectories and catching efficiency are determined for either a mono- or poly-disperse particle distribution which is such that the sum of the volume fractions is equal to one. The catching efficiency computed for the solid 
surface of each bin $\beta_{\text {bin }}$ is corrected for the fraction of particles $\varepsilon_{\text {bin }}$ that sticks to the surface to obtain the catching efficiency of sticking particles:

$$
\beta_{\mathrm{bin}, \text { stick }}=\varepsilon_{\mathrm{bin}} \beta_{\mathrm{bin}}=\varepsilon_{\mathrm{bin}}\left[\frac{\alpha_{\mathrm{bin}} \rho_{p} \vec{u} \cdot \vec{n}}{\mathrm{TWC}\left|\vec{v}_{\infty}\right|}\right]
$$

where $\vec{n}$ is the unit surface normal vector pointing in the direction out of the computational domain and TWC is the total water content. The value of $\varepsilon_{\text {bin }}$ depends on the impact model described in [19]. The computation for the parent bins starts with the bin containing the largest parent particles and the secondary particle mass of each bin is stored in a separate data array. The size of this separate array depends on the number of secondary particle bins, which can be equal to the number of parent bins or equal to a newly defined number of bins. If a parent particle rebounds or shatters the corresponding mass, momentum and energy terms are re-injected in the secondary bin with a secondary particle diameter closest to the one computed with the impact model. This is done such that particle mass flux is conserved.

The calculation for the secondary bin is started from the secondary data array that is imposed as inlet condition on the impingement surface. The far-field values, which were non-zero for the parent bins, are set to zero when computing the secondary cloud. Then the calculation of the Eulerian trajectory of the secondary particles can start. If secondary particles re-impinge further downstream on the surface of the solid geometry, a tertiary particle calculation has to be performed, etc. This post-impact computation was demonstrated numerically for a NACA-0012 airfoil in [21] and for a generic turbofan compressor in [22].

\section{Implementation of Lagrangian (post-)impact method}

In the Lagrangian framework, an impact is detected when the trajectory of a particle intersects a boundary of the computational domain representing a wall. All necessary particle properties are then evaluated at the instant and point of impact defined by this intersection. First, the calculated sticking efficiency at the impact location is compared to a uniformly sampled random number between 0 and 1 in order to decide whether the current particle sticks to the wall or is re-emitted into the flow field.

In case of sticking, the particle is simply removed from the computational domain while its 
properties such as mass, momentum, enthalpy, etc., are stored at the location corresponding to the impinged computational face.

In case of rebound, the velocity vector of the impinging particle is decomposed into a normal and a tangential component whose magnitudes are then modified according to the relations of Table 4 .

In case of shattering, the sum of the masses over the $N_{p}$ re-emitted/secondary particles are set equal to the mass of the impinging particle:

$$
m_{p}=\sum_{i}^{N_{p}} m_{p, i}^{\prime}=\sum_{i}^{N_{p}} w_{p, i}^{\prime} \frac{\pi}{6} \rho_{p, i}^{\prime} d_{p, i}^{\prime 3}
$$

where the superscript ' denotes properties of the re-emitted particles. The physical properties of the re-emitted droplets in Eq. (23), namely $\rho_{p, i}^{\prime}$ and $d_{p, i}^{\prime}$ are given by the wall interaction model. However, determining the respective particle weights $w_{p, i}^{\prime}$ is a matter of statistical sampling. In effect, using a "deterministic" sampling procedure, in which unitary weights are applied to all particles $\left(w_{p, i}=w_{p, i}^{\prime}=1\right.$ for all $\left.i\right)$, is not always feasible as it may induce the generation of a huge number of particles and a corresponding excessive computational cost. In order to obtain these fields at a reasonable expense, homogeneously seeding the entire computational domain with numerical particles appears as important as ensuring similar weights for all particles, as is discussed in section IV A. Since Eq. (23) provides only a single relation to determine the mass of the secondary particles, it may either be assumed that each of these numerical secondary particles carries the same mass (different from the parent particle) or that each secondary particle represents the same (not necessarily integral) "number" of physical particles. In the present case the first option was chosen in order to obtain a faster convergence for the impinging and outgoing mass flux profiles. The size and the sphericity of the secondary particles are then uniformy sampled according to an assumed size distribution while the density is assumed unchanged. Finally, the azimuthal orientation of the tangential velocity vector, allowing to specify the velocity vector of the secondary particles in case of shattering, is chosen via a uniform random procedure.

\section{Results}

A test case has been designed to analyse the behavior of ice particles entering in a engine-like configuration. The geometry consists of a 1.5-stage stator-rotor-stator linear cascade, see Fig. 1, 
Table 5: Geometrical properties.

\begin{tabular}{ccccc}
\hline \hline $\begin{array}{c}\text { Blade ratio } \\
\text { stator } 1: \text { rotor }: \text { stator2 }\end{array}$ & $\begin{array}{c}\text { Rotor } \\
\text { chord }[\mathrm{m}]\end{array}$ & $\begin{array}{c}\text { Rotor height } \\
\text { (hub-tip) }[\mathrm{m}]\end{array}$ & $\begin{array}{c}\text { Rotor } \\
\text { velocity }[\mathrm{m} / \mathrm{s}]\end{array}$ & $\begin{array}{c}\text { Annular hub } \\
\text { radius }[\mathrm{m}]\end{array}$ \\
\hline $16: 20: 25$ & 0.04 & 0.04 & 140 & 10.146 \\
\hline \hline
\end{tabular}

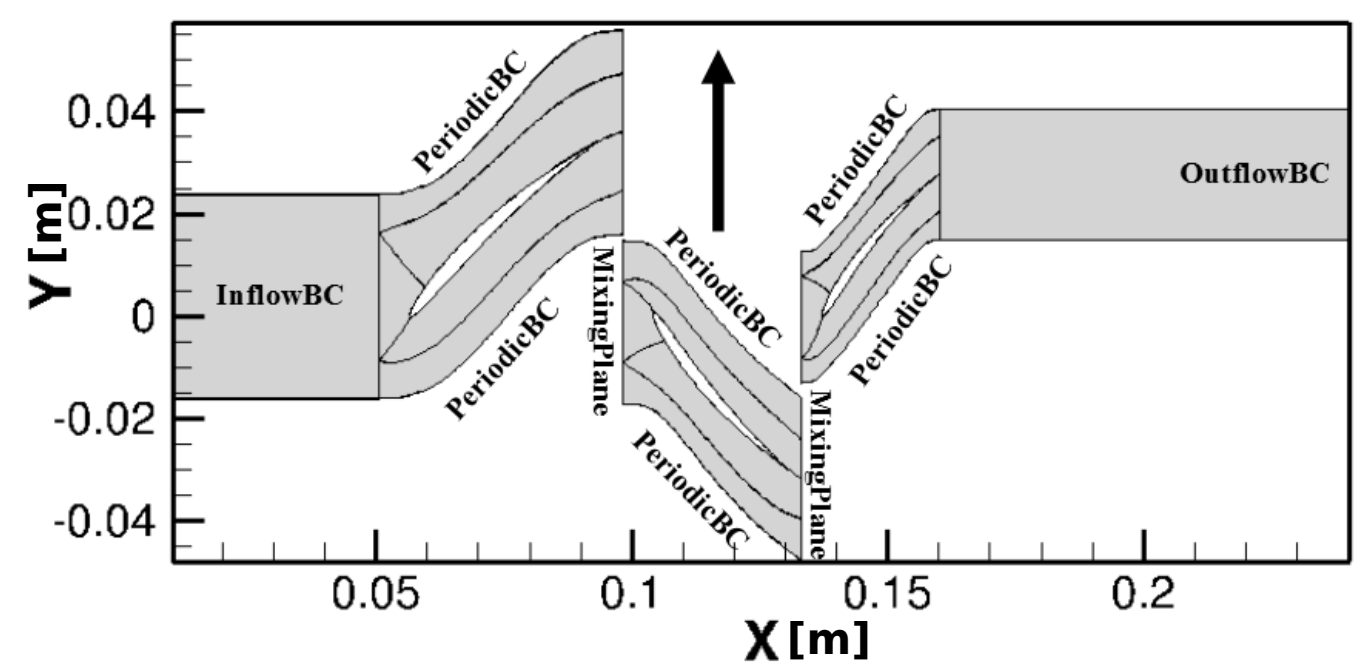

Fig. 1: Schematic generic stator-rotor-stator configuration with imposed boundary conditions.

originally designed at Duke University [50]. A similar configuration was used in the research of, amongst others, Gopinath et al. [51] and Giangaspero [52].

\section{A. Benchmark test case}

For this cascade the ratios of the number of blades equal 16:20:25 (first stator : rotor : second stator) and the chord of the rotor blade is set to $4 \mathrm{~cm}$, see Table 5 . The chords of the first and second stator equal $5 \mathrm{~cm}$ and $3.2 \mathrm{~cm}$, respectively. The annular radius of the cascade is set very large $\left(r / c_{\text {rotor }} \approx 254\right)$ such that the configuration can be considered as a two-dimensional cascade. This accommodates computational methods that cannot handle periodic boundary conditions in the circumferential direction. The rotor moves in positive $y$-direction at a velocity of $140 \mathrm{~m} / \mathrm{s}$. The grid is linearly cascaded using periodic boundary conditions and in $z$-direction the hub and casing are solid walls. The sliding interfaces between the first stator and the rotor and that between the rotor and the second stator are represented as mixing planes, which allows a steady-state analysis of the flow through the configuration, see Fig. 1. 
Table 6: Aerodynamic conditions.

\begin{tabular}{cccccc}
\hline \hline $\begin{array}{c}\text { Mach } \\
M_{\infty}\end{array}$ & $\begin{array}{c}\text { Incidence } \\
\text { angle }\left[{ }^{\circ}\right]\end{array}$ & $\begin{array}{c}\text { Total pressure } \\
p_{\text {tot }}[\mathrm{Pa}]\end{array}$ & $\begin{array}{c}\text { Total temperature } \\
T_{\text {tot }}[\mathrm{K}]\end{array}$ & $\begin{array}{c}\text { Relative } \\
\text { humidity }[\%]\end{array}$ & $\begin{array}{c}\text { Exhaust static } \\
\text { pressure } p[\mathrm{~Pa}]\end{array}$ \\
\hline 0.25 & 53.5 & 37850 & 296 & 25 & 40472 \\
\hline \hline
\end{tabular}

The grid applied in the numerical simulations is a multi-block structured grid consisting of $8,320,6,592$ and 8,320 cells for stator 1 , rotor and stator 2 , respectively. The inlet block contains 1,344 control volumes and the outlet block contains 3,840 control volumes. The obtained solution (e.g. catching efficiency) on this grid resolution is checked to be grid-independent in the majority of the domain. Only in regions with sharp gradients some grid dependency is still observed, see section IV E.

\section{B. Aerodynamic conditions}

The air flow field is obtained employing the Euler equations for steady inviscid flow. At the inlet the stagnation pressure, stagnation temperature and the velocity vector are prescribed and at the outlet the static pressure is prescribed, see Table 6 . The direction of the inlet velocity is set at an incidence angle of $53.5^{\circ}$, which represents, for instance, a fan upstream of the first stator. The aerodynamic field is computed for a pressure level of $p_{t o t}=37,850 \mathrm{~Pa}$. The relative humidity $\mathrm{RH}$ is set to $25 \%$. In the Eulerian framework this parameter will only affect the mass transfer along the particle trajectories, since the air flow field is computed for dry conditions. For the Lagrangian simulations a separate mass conservation equation is solved for the vapor species.

The aerodynamic conditions are set such that at least some ice particle melting is to be expected. The pressure coefficient $c_{p}$ and the air temperature $T_{a}$ obtained with the in-house flow solvers CEDRE and MooseMBFlow used for the Lagrangian and Eulerian ice crystal trajectory simulations, respectively, are compared in Fig. 2. From this plot it can be seen that the agreement for the surface distribution of $c_{p}$ is very good. The surface distributions for the air temperature $T_{a}$ are, however, slightly different, i.e. with a maximum difference of less than $0.5 \mathrm{~K}$ on the suction side, with CEDRE's values above those of MooseMBFlow. This difference increases with increasing distance from the compressor inlet. In order to eliminate these slight discrepancies, the same aerodynamic 

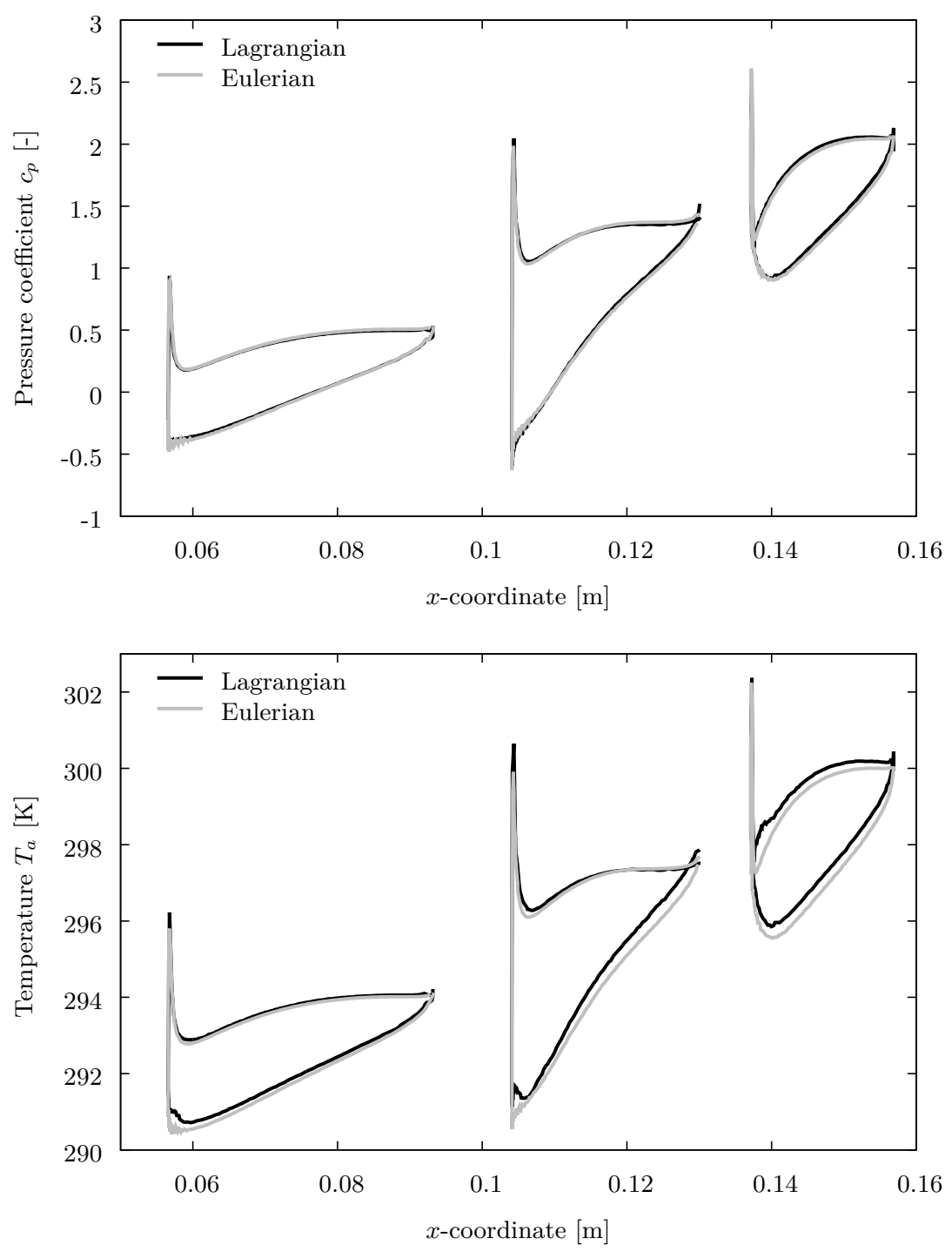

Fig. 2: Distributions surface pressure coefficient $c_{p}$ (top) and air temperature $T_{a}$ (bottom).

flow field could have been used for the Eulerian as well as for the Lagrangian simulations of the dispersed phase. However, this would have required an identical input/ output format for the aerodynamic solution fields, which turned out to be unavailable. Moreover, the agreement between the aerodynamic flow fields was found sufficiently close to warrant meaningful comparisons between the results from the dispersed phase solvers, as is demonstrated below.

\section{Conditions for dispersed phase}

The particles are represented as isometric spheroids with a sphericity of either $\Phi=0.7$ (run a and b) or $\Phi=1$ (run c), see Table 7 . The particles enter the compressor stage at an initial 
Table 7: Particle properties.

\begin{tabular}{ccccccc}
\hline \hline $\begin{array}{c}\text { Run } \\
\#\end{array}$ & $\begin{array}{c}\text { Volume-equivalent } \\
\text { diameter } d_{p}[\mu \mathrm{m}]\end{array}$ & $\begin{array}{c}\text { Sphericity } \\
\Phi\end{array}$ & $\begin{array}{c}\text { Crosswise } \\
\text { sphericity } \Phi_{\perp}\end{array}$ & $\begin{array}{c}\text { Temperature } \\
T_{p}[\mathrm{~K}]\end{array}$ & $\begin{array}{c}\text { IWC } \\
{\left[\mathrm{kg} / \mathrm{m}^{3}\right]}\end{array}$ & $\begin{array}{c}\text { Density } \\
\rho_{i}\left[\mathrm{~kg} / \mathrm{m}^{3}\right]\end{array}$ \\
\hline $\mathrm{a}$ & 50 & 0.7 & 0.7 & 268 & 0.002 & 917 \\
$\mathrm{~b}$ & 20 & 0.7 & 0.7 & 268 & 0.002 & 917 \\
$\mathrm{c}$ & 50 & 1.0 & 1.0 & 268 & 0.002 & 917 \\
\hline \hline
\end{tabular}

temperature of $-5^{\circ} \mathrm{C}$ and the initial velocity of the particles equals the inlet air velocity. The results for two different volume-equivalent particle sizes will be shown: $d_{p}=50 \mu \mathrm{m}$ (run a) and $d_{p}=20$ $\mu \mathrm{m}$ (run b). For validation and comparison purposes, one set of computations is performed with a deactivated impact model, i.e. all particles are assumed to stick to the surface. The other set of computations is performed by assuming partial deposition according to the impact model described in section III. Imposing the boundary conditions for the Eulerian solver is very straightforward and very similar to that for the carrier phase. At the mixing plane interface the conservative variables for the dispersed phase are again averaged in circumferential direction. This is not the case for the Lagrangian solver, for which particular care needs to be taken when injecting the particles into the next stage.

In the Lagrangian simulations, $5 \cdot 10^{5}$ particles are injected at the inflow boundary condition and this target number is kept constant when they are re-injected into the rotor and second stator stages according to the averaged properties of the dispersed phase calculated at the mixing plane. At the inlet the particles are positioned such that the distance $h$ between the particles is identical. This point is crucial insofar as a homogeneous seeding was found to drastically improve convergence. This behavior may be understood through an analogy with numerical integration. Assume that the particles are positioned on a segment $[a, b]$ in such a way that they are separated by a constant spacing $h=(b-a) / N$, with $N$ the number of particles. Furthermore, assume that their weights $w_{p}$ (see Eq. 23) are imposed such that they match a mass flux distribution given by a function $w_{p, i}=f\left(x_{i}\right)$. Then, applying the trapezoidal rule at the particle positions to approximate the 
resulting mass flux yields:

$$
\int_{a}^{b} f(x) d x \approx \frac{h}{2}[f(a)+2 f(a+h)+2 f(a+2 h) \ldots+f(b)]=\sum_{i=1}^{N} k_{i} w_{p, i},
$$

with $k_{i}=h / 2$ for $i=1, i=N$ and $k_{i}=h$ otherwise. This approximation yields a quadratic convergence rate with respect to the spacing $h \sim 1 / N$. In contrast, if the position of the $n$ particles on the segment $[a, b]$ is randomly sampled as in standard Monte-Carlo approaches, the convergence rate of the numerical integration given by Eq. (24) will drop to $1 / \sqrt{N}$ according to the central limit theorem. Although simplified, the above reasoning helps to understand how homogeneous particle seeding may significantly improve convergence in Lagrangian simulations. Also, this reasoning naturally applies to all other phase space variables of the spray density function, such as size, velocity, temperature, etc. Clearly, enforcing uniform spatial seeding is straightforward when injecting particles on planes. However, it is much more complicated on curved surfaces, such as airfoils, where the impinging particles need to be re-emitted when using a realistic particle wall interaction model. Such procedure is not available in the Lagrangian solver yet and it remains to be shown how much it could alleviate convergence issues.

A complementary strategy to limit convergence issues could consist in the use of a mollification procedure when projecting Lagrangian data on the Eulerian grid. With this type of approach, the influence of a particle on the Eulerian averages is not only accounted for in the cell where the particle is located, but also in a set of neighboring cells [53]. From a theoretical point of view, this mollification step may be seen as a regularization of the Dirac delta functions used to approximate the spray density function. The implementation of such mollification procedure is currently being investigated. In the meantime, in a post-processing step remaining oscillations are damped by simply applying a diffusion equation to the resulting mass flux profiles:

$$
\frac{\partial \dot{m}}{\partial t}-\nabla \cdot \frac{\epsilon^{2}}{\tau} \nabla \dot{m}=0
$$

here $\epsilon$ and $\tau$ are characteristic length and time scales, respectively, of the diffusion process. $\epsilon$ is set to a local characteristic curvilinear length scale $l_{s}$. Eq. (25) is then solved with an explicit first-order time advancement with the time scale $\tau=\omega \Delta t$ linked to the CFL stability condition for its numerical resolution. It is well-known that this stability condition is very restrictive for an 

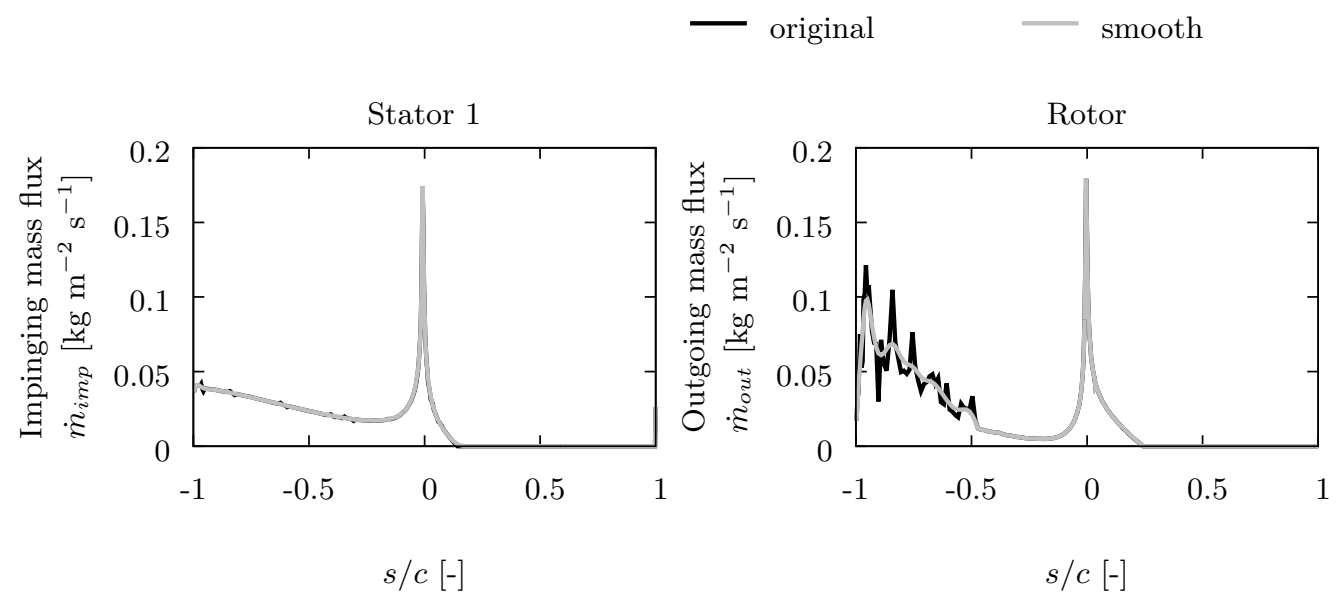

Fig. 3: Diffusion procedure applied to raw Eulerian fields obtained for run a from Lagrangian simulations for full deposition (left) and for partial deposition mode (right).

explicit time advancement. However, the cost remains acceptable since only high wave number oscillations are to be damped. Finally, the diffusion equation is deactivated according to minimum and maximum threshold values $\dot{m}_{l o w}$ and $\dot{m}_{u p}$ with respect to the peak mass flux. This ensures that both the peak values and the impingement boundaries do not become overly smeared. These values were set to $\dot{m}_{\text {low }}=0.1 \dot{m}_{\max }$ and $\dot{m}_{\text {up }}=0.9 \dot{m}_{\max }$, respectively, in the present case. Figure 3 illustrates raw and smoothed profiles for impinging mass fluxes (full deposition simulation) and outgoing mass fluxes (partial deposition model). The discussion and analysis of these results are provided in sections IVD and IVE. For the moment, the sole focus is on the illustration of the post-processing procedure for the Lagrangian results. It appears that the impinging mass flux profile displays only very slight oscillations on the left of Fig. 3 so that the diffusion procedure has hardly any effect. On the contrary, very significant oscillations occur for the outgoing mass flux profiles, as illustrated on the right side of Fig. 3. Here, five iterations of the diffusion equation are necessary to obtain a smooth curve. Clearly, the amount of diffusion applied is somewhat arbitrary since a scale separation between physically meaningful variations and oscillations due to a lack of convergence cannot easily be established a posteriori. Therefore, it must be emphasized for later analysis that the Lagrangian results for the outgoing mass flux profiles possess important uncertainties on a large portion of the pressure sides of the first stator and the rotor. 

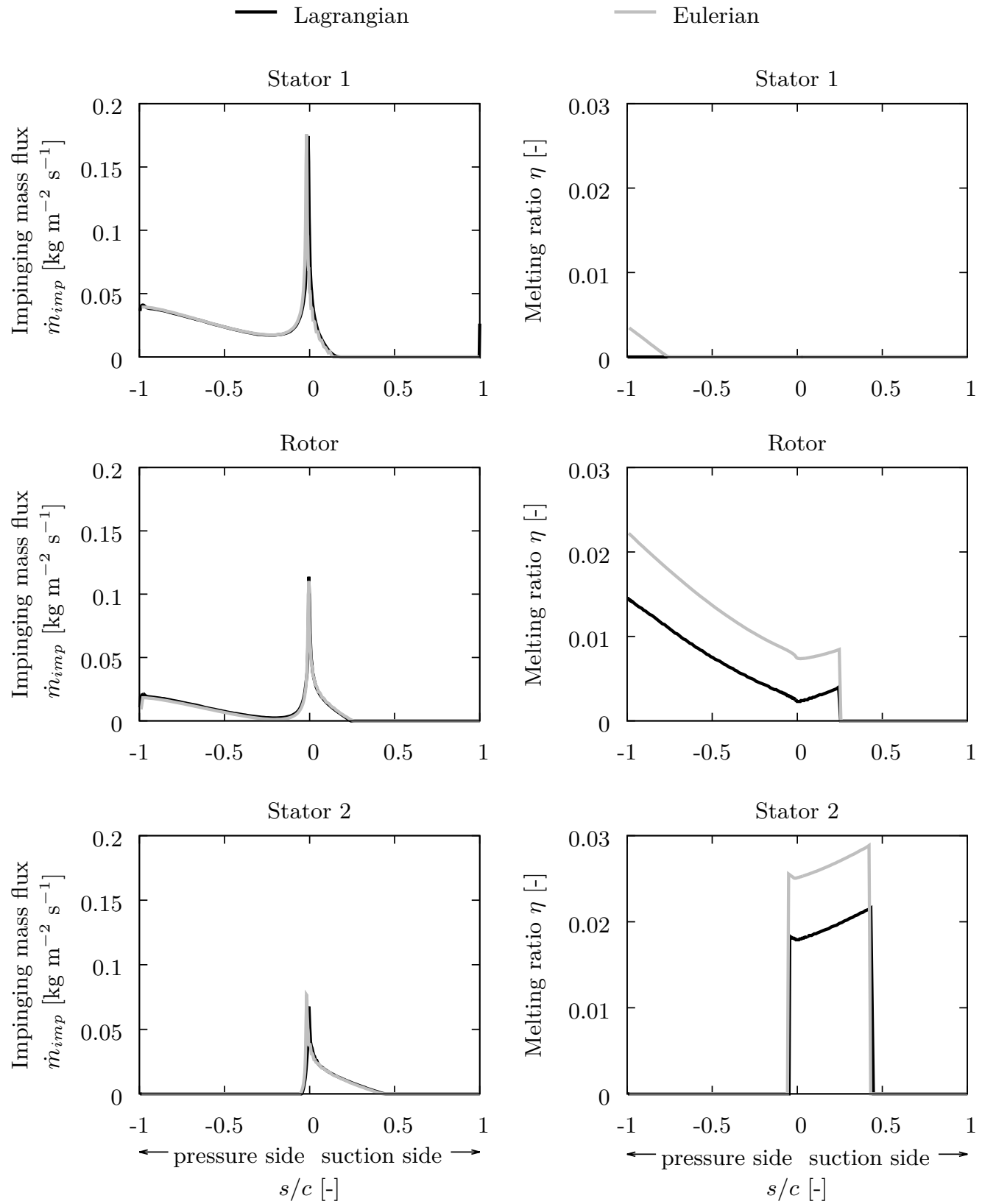

Fig. 4: Full deposition, run a $\left(d_{p}=50 \mu \mathrm{m}, \Phi=0.7, \Phi_{\perp}=0.7\right)$ : Distribution of impinging mass flux $\dot{m}_{i m p}$ (left) and melting ratio $\eta$ (right) on blade surfaces.

\section{Lagrangian vs. Eulerian: Full deposition mode}

Figures 4,5 and 6 present the surface distributions of the impinging mass flux $\dot{m}_{i m p}$ (left) and the melting ratio $\eta$ (right) along the blade surfaces. For the results obtained here it is assumed that the (partially melted) ice crystals fully deposit on the surface. From the surface distributions of $\dot{m}_{i m p}$ it is seen that, in general, particles impact: 

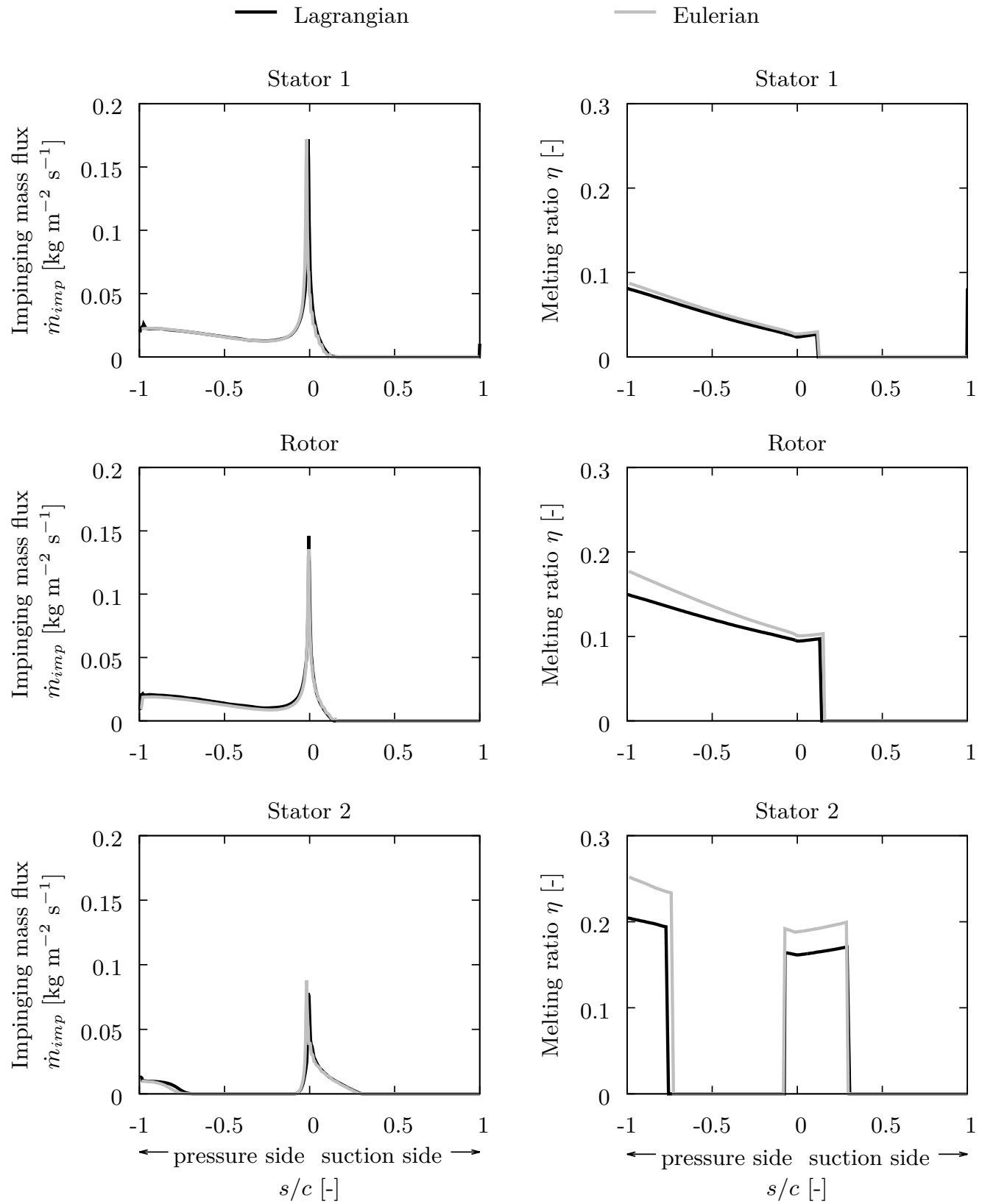

Fig. 5: Full deposition, run b $\left(d_{p}=20 \mu \mathrm{m}, \Phi=0.7, \Phi_{\perp}=0.7\right)$ : Distribution of impinging mass flux $\dot{m}_{i m p}$ (left) and melting ratio $\eta$ (right) on blade surfaces.

- on the pressure side of the first stator,

- on the pressure side of the rotor,

- on the suction side of the second stator.

In case of run b, a fraction of the small particles are able to follow the curvature of the blades of the second stator and impinge on the pressure side close to the trailing edge. The majority of the large 

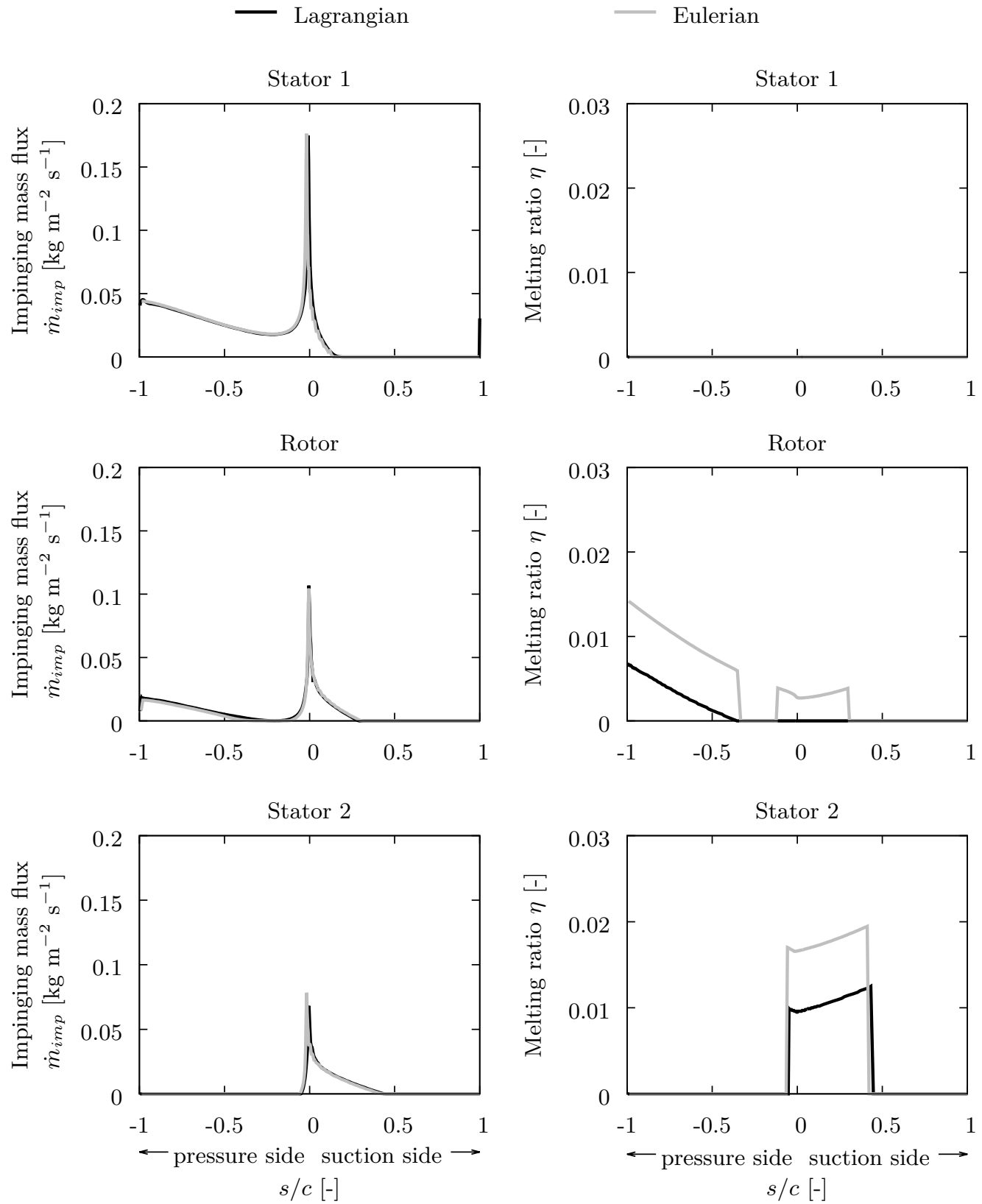

Fig. 6: Full deposition, run c $\left(d_{p}=50 \mu \mathrm{m}, \Phi=1.0, \Phi_{\perp}=1.0\right)$ : Distribution of impinging mass flux $\dot{m}_{i m p}$ (left) and melting ratio $\eta$ (right) on blade surfaces.

particles $\left(d_{p}=50 \mu \mathrm{m}\right)$ impinge on the first stator and only a small portion impacts the second stator or leaves the cascade without impact. For the small particles with $d_{p}=20 \mu \mathrm{m}$ the results for the catching efficiency on the first stator and on the rotor are almost equal. Similarly, the comparison of the results of run a and those for run c indicate that for all these stages particle sphericity $\Phi$ has a negligible effect on the distribution of the impinging mass flux distribution. Furthermore, the peak 
catching efficiency in the region near the leading edge decreases from approximately $0.18 \mathrm{~kg} / \mathrm{m}^{2} \mathrm{~s}$ for the first stator to approximately $0.073 \mathrm{~kg} / \mathrm{m}^{2} \mathrm{~s}$ for the second stator. The agreement between the results for $\dot{m}_{i m p}$ obtained from the Lagrangian and the results from the Eulerian method is very good.

The melting ratio $\eta$ of small particles (run b) is much higher than the melting ratio of the larger particles (runs a and c). On the contrary, the effect of particle sphericity variations on melting appears relatively moderate. It should be noted that the melting ratio range plotted in Fig. 5 is ten times larger than the range plotted in Fig. 4 and Fig. 6 in order to increase visibility. The 50 $\mu \mathrm{m}$-diameter particles have an average melting ratio of $0,0.01$ and 0.02 for stator 1 , the rotor and stator 2 , respectively. For the particles with $d_{p}=20 \mu \mathrm{m}$ these values are $0.08,0.14$ and 0.2 for stator 1 , the rotor and stator 2 , respectively. The differences in melting ratio of the results from the Eulerian method and of those from the Lagrangian method are more significant. In general, $\eta$ obtained from the Eulerian method is higher than $\eta$ obtained from the Lagrangian method and this

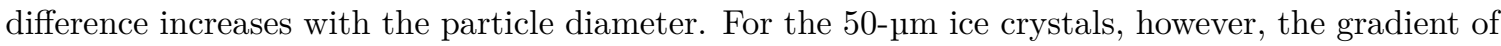
the distribution of the melting ratio along the blade surface is almost equal for both methods, while this is not quite so for the $20-\mu \mathrm{m}$ ice crystals. It is assumed that the differences are a consequence of the slight temperature differences between the aerodynamic flow fields and discrepancies in the evaluation of thermophysical properties of the carrier phase for particle heat and mass transfer, see section IIB.

\section{E. Lagrangian vs. Eulerian: Partial deposition mode}

In the second set of simulations the full impact model described in Section III is applied. In the Eulerian simulation the secondary cloud of rebounded or shattered particles is distributed over five bins consisting of different particle sizes: $50 \mu \mathrm{m}, 40 \mu \mathrm{m}, 30 \mu \mathrm{m}, 20 \mu \mathrm{m}$, and $10 \mu \mathrm{m}$. However, the periodic boundaries in the linear cascade introduce the possibility of crossing trajectories, which causes the computation to diverge. This trajectory crossing is purely numerical as particle trajectories are almost never expected to cross in icing applications due to the very low particle volumetric concentrations encountered. In the numerical method crossing of particle trajectories is prevented by introducing additional bins that handle the pressure and suction surface of each 
blade separately. So, instead of $N$ secondary bins, now $6 N$ secondary bins are to be solved for. Re-impingement of rebounding or secondary particles caused by shattering from an upstream blade row is taken into account in a new iteration of the secondary cloud. Another problem arises by the impact model itself. At the leading edge of a blade it may happen that the secondary particles get a large negative velocity in $x$-direction and/or a large velocity in $y$-direction. When this occurs the secondary particles can either cross trajectories in front of the blade or they can move even further upstream back into the mixing plane. To avoid this the secondary particle velocity in $x$-direction is set to a minimum value of $0 \mathrm{~m} / \mathrm{s}$, the same velocity correction being applied in the Lagrangian method for consistency. Although adapting the physical model is not a preferred solution, it only affects a small fraction of secondary particles that rebounds from the leading edge of the blades.

The impinging mass flux $\dot{m}_{i m p}$ and the outgoing mass flux $\dot{m}_{\text {out }}$ are presented in Fig. 7 on the left and right side, respectively, for run a. When the results for the impinging mass flux for partial deposition are compared to the ones for full deposition, see Fig. 4, it is seen that particles or particle fragments re-impinge close to the trailing edge on the pressure side of the first stator and on the blades further downstream in the cascade. Furthermore, it can be seen that a fraction of the particles sticks to the surface, since the impinging mass flux is larger than the outgoing mass flux. This sticking effect is most clearly seen for the second stator. In the case of partial deposition the difference between the results from the Lagrangian method and the ones from the Eulerian method is significant. For the Eulerian method a large fraction of the ice crystals rebounds near the trailing edge at the pressure side of the first stator and of the rotor. This leads to much higher mass fluxes further downstream in the cascade compared to the situation for the Lagrangian method, which suffers from large uncertainties due to lack of convergence in the same area, as detailed in section IV C.

The distribution of the melting ratio along the blade surfaces is given in Fig. 8. Note that the range for the melting ratio increases from left to right by a factor 10 (rotor) and 100 (stator 2). From the comparison of the results from the Lagrangian method and the ones from the Eulerian method it is seen that different outcomes are also obtained for the particle melting ratio, which can be ascribed to a number of factors. First, it is obvious that the multiple re-impingements at the trailing edge 
Lagrangian

Stator 1

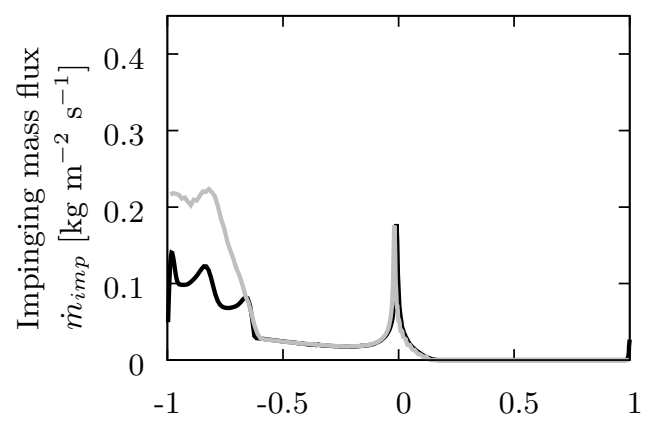

Rotor

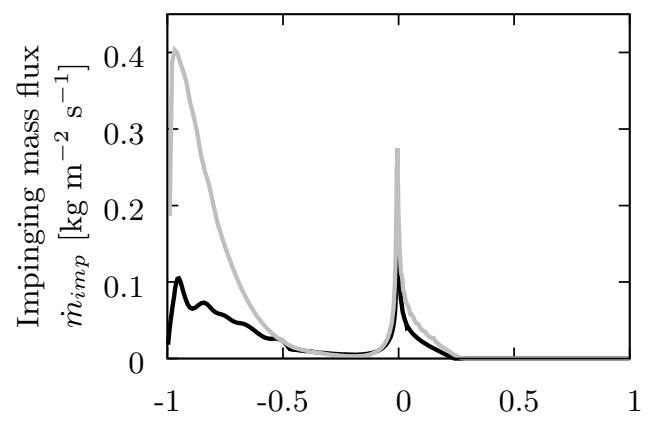

Stator 2

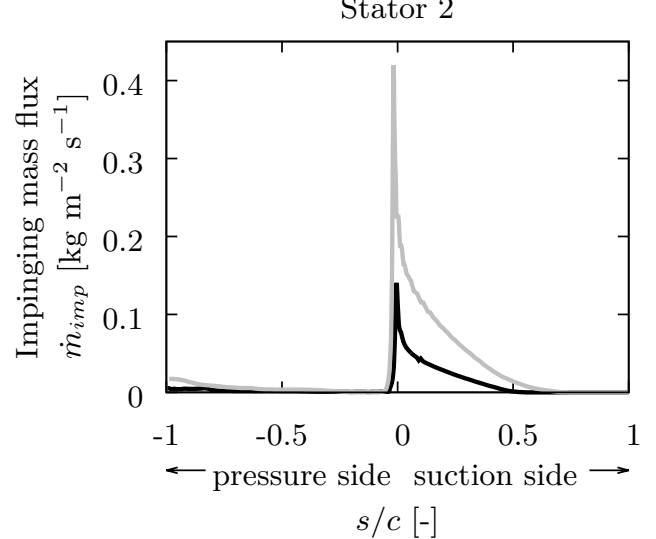

Stator 1

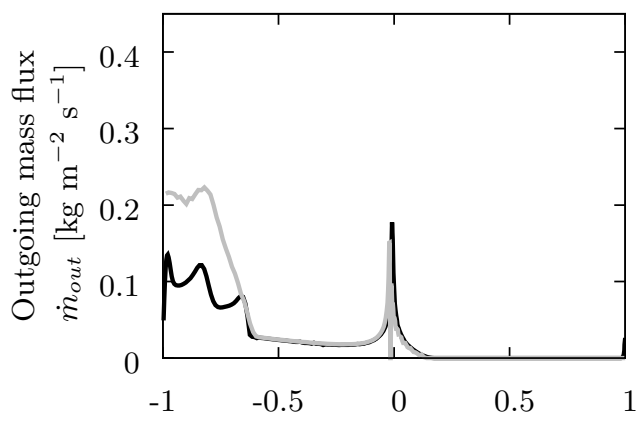

Rotor

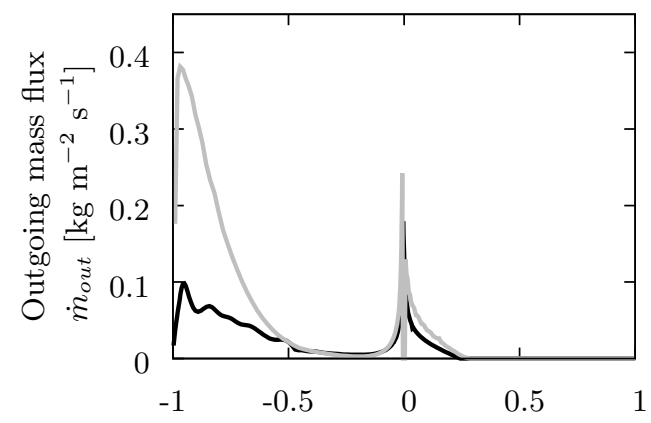

Stator 2

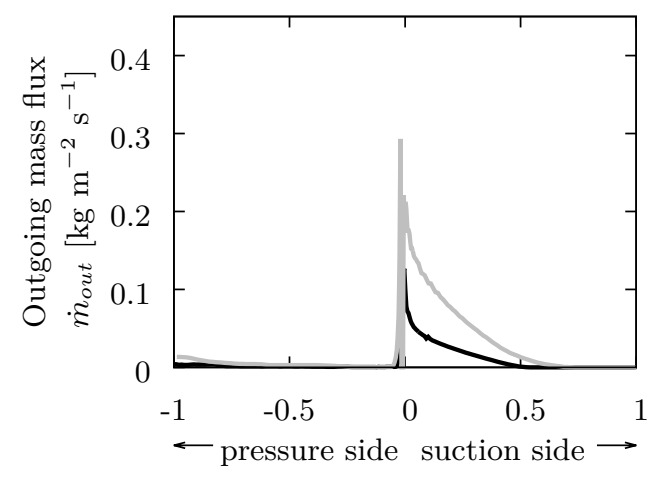

$s / c[-]$

Fig. 7: Partial deposition, run a $\left(d_{p}=50 \mu \mathrm{m}, \Phi=0.7, \Phi_{\perp}=0.7\right)$ : Distribution of impinging mass flux $\dot{m}_{\text {imp }}$ (left) and outgoing mass flux $\dot{m}_{\text {out }}$ (right) on blade surfaces.

of the blades in case of the Eulerian method results in different melting ratios compared to the Lagrangian method as a consequence of the different trajectories that the secondary particles follow in that region. Secondly, the difference can be explained partly by the discretization used for the bins in the Eulerian approach. For instance, particles that are supposed to rebound with a diameter of 48 $\mu \mathrm{m}$ are re-injected as part of the bin containing $50 \mu \mathrm{m}$ particles, while in the Lagrangian approach 
Lagrangian

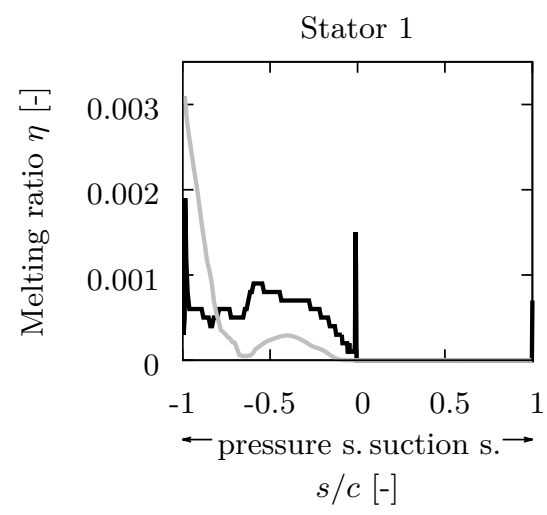

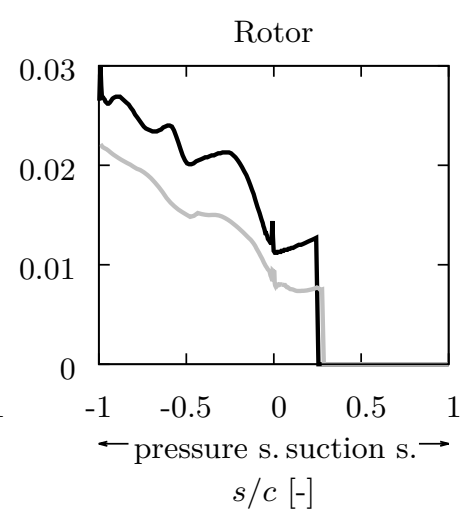

Fig. 8: Partial deposition, run a $\left(d_{p}=50 \mu \mathrm{m}, \Phi=0.7, \Phi_{\perp}=0.7\right)$ : Distribution of melting ratio $\eta$ on the surface of each blade row in compressor.

the prescribed distribution of particle diameters is preserved. Since a fraction of the ice crystals are fully rebounding, and sublimate or evaporate along their trajectory, the Eulerian discretization should result in a lower melting ratio compared to the result of the Lagrangian approach. This is true at least for the melting ratio along the surface of the first stator and along the surface of the rotor. However, the melting ratios are enhanced due to fragmentation, compare Fig. 8 to Fig. 4 (right). This results in the melting ratio for the Eulerian method becoming larger than that for the Lagrangian method further downstream in the cascade, as was the case for full deposition. Finally, the Eulerian approach displays significant numerical dissipation at sharp gradients in the particle density field due to the used flux scheme and reconstruction limiter. For this test case ice crystal impingement results in a number of closely packed particle trajectories that dissipate considerably. It is expected that this feature is most visible for the melting ratio at the pressure surface of the second stator, for which the melting ratio obtained with the Eulerian method is much higher than the one obtained with the Lagrangian method.

\section{F. Lagrangian vs. Eulerian: Problems encountered}

Despite the application of a special post-impact method for the Eulerian approach, that handles crossing trajectories as a consequence of periodic boundary conditions, numerically the crossing of trajectories that originate from concave surfaces could not be avoided. The slightly concave surface 
at the pressure side of the blades results in converging particle trajectories. In the Eulerian method the resulting averaged trajectory is forced back towards the airfoil surface. This leads to multiple rebound or shattering events, which are not seen in the results from the Lagrangian method. In order to show the effect of the concave surface, the Eulerian method is repeated for run a in which the first stator has been adapted to have a non-concave, straight surface near the trailing edge of the pressure side. In the left graph of Fig. 9 the distribution of the surface pressure coefficient $c_{p}$ of the aerodynamic flow field around the stator vane with a concave surface (black line) and the one with a straight surface (grey line) are presented. The difference in aerodynamic conditions near the pressure surface leads to a slightly different impinging mass flux over most of the surface, see the right side of Fig. 9. However, the largest difference is the absence of the peak in the distribution of $\dot{m}_{i m p}$ on the pressure surface near the trailing edge, which demonstrates that particle re-impingement does not happen in case of a straight (or convex) surface.

As far as the Lagrangian approach is concerned, specific pre- and post-processing steps, namely the equidistant distribution of particle injection points at the inlet plane as well as on the mixing planes on the one hand and a diffusion procedure applied to the resulting fields on the other (see sec. IV C) appeared necessary to obtain satisfactory quantitative results for the impinging and outgoing mass fluxes. Despite these efforts, important uncertainties remained for the outgoing mass fluxes on a significant portion of the pressure side for the first stator and for the rotor. Moreover, these procedures were tailored to the present simplified test case and extensions applicable to more realistic configurations need to be developed. Finally, these procedures may prove insufficient to alleviate the convergence issues inherent to the Lagrangian approach to such configurations.

\section{Conclusion}

This study presented a detailed comparison between an Eulerian and a Lagrangian approach for the numerical simulation of melting ice crystal trajectories and impacts in an engine-like configuration. When particles were assumed to fully deposit on the blade surfaces, impinging mass flux profiles obtained with both approaches were in excellent agreement. Some disagreement was observed for the melting ratio, which could be ascribed to the different modeling of the air flow and slightly different transport properties for the vapor species used by the two methods. On the basis 


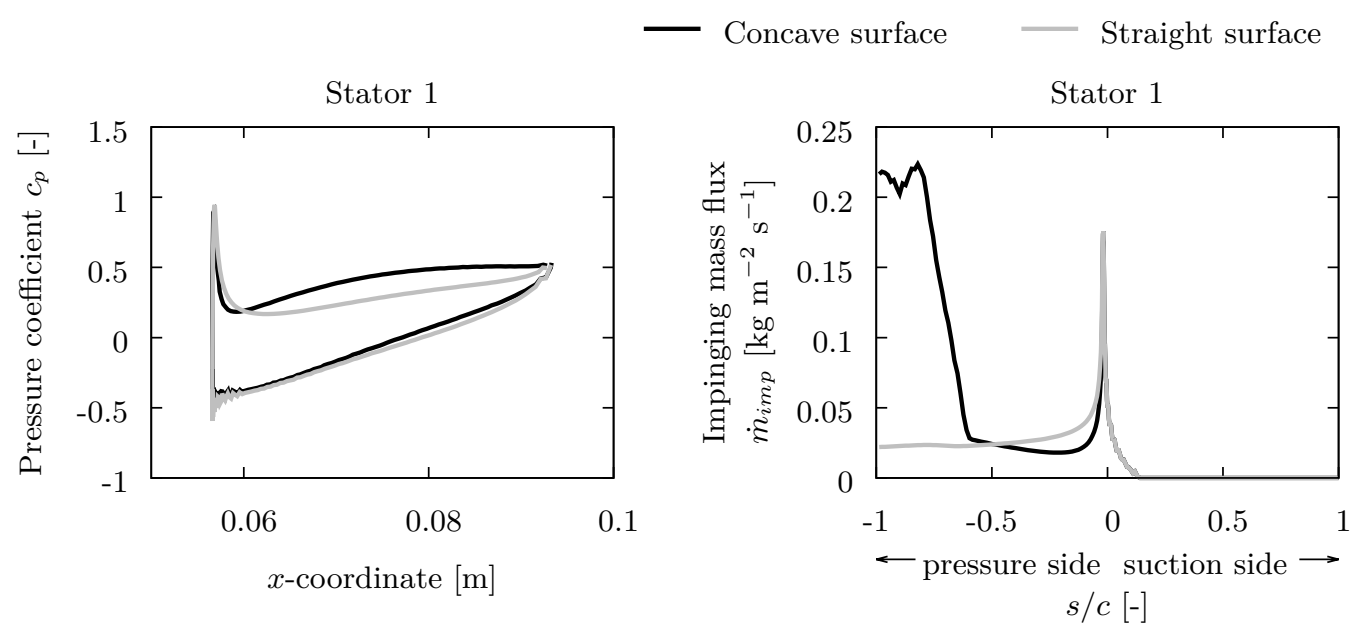

Fig. 9: Distribution of pressure coefficient $c_{p}$ (left) and impinging mass flux $\dot{m}_{i m p}$ (partial deposition, right) obtained for run a with the Eulerian method for stator 1.

of this agreement, it could be interesting to compare the present results with those of other numerical solvers dedicated to ice crystal trajectory simulations in engine-like configurations. In addition, variations of parameters such as particle sphericity and diameter were performed to evaluate their effect on melting and evaporation for the full deposition case. The impinging mass flux distributions appeared to change slightly with varying particle size while they were almost insensitive to variations in sphericity, at least for the range of values explored here. As far as melting is concerned, the effect of variations of particle size was very significant and appeared largely predominant over shape variations.

When a realistic model predicting rebound, shattering or sticking as possible outcomes of particle impacts on the blades was used, differences between both approaches became more pronounced. On the one hand, the Eulerian method displayed some difficulties in handling concave surfaces due to emanating crossing trajectories, which lead to a disagreement in the results. Further downstream in the cascade the averaging over the mixing planes enhanced the differences. On the other hand, the Lagrangian approach struggled to produce converged fields. The convergence behavior was partly improved through an injection approach ensuring homogeneous spatial seeding and the application of a diffusion procedure as a post-processing step. However, important uncertainties remained on portions of the pressure side of the first stator and rotor for the outgoing mass flux, precisely at 
locations where the disagreement between both approaches was the most significant. In addition, the specific pre- and post-processing steps applied to the Lagrangian results took advantage of the present simplified configuration and further developments are necessary to generalize them.

In summary, it appears that standard Eulerian and Lagrangian approaches encounter some of their known limitations when handling a linear cascade, i.e. the description of crossing trajectories in the Eulerian approach versus convergence issues for the Lagrangian approach. These limitations need to be addressed through additional research efforts to render numerical simulations of realistic engine configurations feasible. In addition, the aforementioned trajectory/ impingement models need to be coupled to an accretion module to perform numerical simulations of icing phenomena. Thus, extensions of the impingement models to account for the interaction between the melting ice crystals and the freezing liquid films are necessary. Furthermore, particle/ ice reemision due to liquid film atomization via aerodynamic or centrifugal forces needs to be taken into account. Once these phenomena are appropriately modeled and coupled within a 3D icing simulation suite, the capacity of the latter to qualitatively predict the occurence of accretion depending on flight regimes and exterior conditions may be assessed. These different aspects are the subject of ongoing work. Obtaining reliable experimental data on ice crystal melting ratios and ice layer thicknesses in engine like configurations seems very challenging and additional efforts are necessary if quantitative validations of numerical tools are to be performed on realistic engine configurations in the future.

\section{Funding}

This work received funding from the European Union's Seventh Framework Programme for research, technological development and demonstration under grant agreement n ACP2-GA-2012314314. The development of CEDRE's Lagrangian solver for engine icing applications also received funding from Safran Aircraft Engines (SAE).

\section{Acknowledgements}

The aforementioned fundings from the European Union within the Seventh Framework Programme for research, technological development and demonstration under grant agreement $n^{\circ} \mathrm{ACP} 2$ GA-2012-314314 and from Safran Aircraft Engines (SAE) are gratefully acknowledged. 
The second author would like to warmly thank Valentin Biasi and Ysolde Prévereaud for their assistance in the set-up of the aerodynamic simulations and Anthoine Biancherin for his invaluable support in the development of CEDRE's Lagrangian solver.

\section{References}

[1] Bravin, M., Strapp, J. W., and Mason, J., "An Investigation into Location and Convective Lifecycle Trends in an Ice Crystal Icing Engine Event Database," Society of Automotive Engineers, Paper 201501-2130, Jun 2015, doi: 10.4271/2015-01-2130.

[2] Wright, W. B., Struk, P., Bartkus, T., and Addy, G., "Recent Advances in the LEWICE Icing Model," Society of Automotive Engineers, Paper 2015-01-2094, Jun 2015, doi: 10.4271/2015-01-2094.

[3] Bourgault, Y., Boutanios, Z., and Habashi, W. G., "Three-Dimensional Eulerian Approach to Droplet Impingement Simulation using FENSAP-ICE, Part 1: Model, Algorithm, and Validation," Journal of Aircraft, Vol. 37, No. 1, 2000, pp. 95-103, doi: 10.2514/2.2566.

[4] Aliaga, C. N., Aubé, M. S., Baruzzi, G. S., and Habashi, W. G., "FENSAP-ICE-Unsteady: Unified In-Flight Icing Simulation Methodology for Aircraft, Rotorcraft, and Jet Engines," Journal of Aircraft, Vol. 48, No. 1, 2011, pp. 119, doi: 10.2514/1.C000327.

[5] Villedieu, P., Trontin, P., Guffond, D., and Bobo, D., "SLD Lagrangian Modeling and Capability Assessment in the Frame of ONERA 3D Icing Suite," AIAA Paper 2012-3132, Jun 2012, doi: 10.2514/6.20123132.

[6] Trontin, P., Blanchard, G., Kontogiannis, A., and Villedieu, P., "Description and Assessment of the New ONERA 2D Icing Suite IGLOO2D,” AIAA Paper 2017-3417, Jun 2017, doi: 10.2514/6.2017-3417.

[7] Dezitter, F., Grandin, A., Brenguier, J.-L., Hervy, F., Schlager, H., Villedieu, P., and Zalamansky, G., "HAIC-High Altitude Ice Crystals," AIAA Paper 2013-2674, Jun 2013, doi: 10.2514/6.2013-2674.

[8] Sanjosé, M., Senoner, J.-M., Jaegle, F., Cuenot, B., Moreau, S., and Poinsot, T., "Fuel Injection Model for Euler-Euler and Euler-Lagrange Large-Eddy Simulations of an Evaporating Spray inside an Aeronautical Combustor," International Journal of Multiphase Flow, Vol. 37, No. 5, 2011, pp. 514-529, doi: 10.1016/j.ijmultiphaseflow.2011.01.008.

[9] Tseng, C.-C. and Hu, H.-A., "Flow Dynamics of a Pitching Foil by Eulerian and Lagrangian Viewpoints," AIAA Journal, Vol. 52, No. 2, 2015, pp. 712-727, doi: 10.2514/1.J053619.

[10] Sun, D., Garmory, A., Page, G. J., and Tristanto, I., "Simulation of Particle Flow in Inertial Particle Separators with Eulerian VR-QMOM Method," AIAA Journal, Vol. 54, No. 12, 2016, pp. 3803-3812, 
doi: $10.2514 / 1 . J 054981$.

[11] Williams, F. A., "Spray Combustion and Atomization," Physics of Fluids, Vol. 1, No. 6, 1958, pp. 541545, doi: $10.1063 / 1.1724379$.

[12] Laurent, F. and Massot, M., "Multi-fluid Modelling of Laminar Polydisperse Spray Flames: Origin, Assumptions and Comparison of Sectional and Sampling Methods," Combustion Theory and Modelling, Vol. 5, No. 4, 2001, pp. 537-572, doi: 10.1088/1364-7830/5/4/303.

[13] Desjardins, O., Fox, R. O., and Villedieu, P., "A Quadrature-Based Moment Method for Dilute Fluid-Particle Flows," Journal of Computational Physics, Vol. 227, No. 4, 2008, pp. 2514-2539, doi: 10.1016/j.jcp.2007.10.026.

[14] Refloch, A., Courbet, B., Murrone, A., Villedieu, P., Laurent, C., Gilbank, P., Troyes, J., Tessé, L., Chaineray, G., and Dargaud, J., "CEDRE Software," AerospaceLab, , No. 2, 2011, pp. 1-10.

[15] Murrone, A. and Villedieu, P., "Numerical Modeling of Dispersed Two-Phase Flows," AerospaceLab, , No. 2, 2011, pp. 1-13.

[16] Hospers, J. M., Eulerian Method for Super-Cooled Large-Droplet Ice-Accretion on Aircraft Wings, Ph.D. thesis, University of Twente, Enschede, the Netherlands, 2013, doi: 10.3990/1.9789036536172.

[17] Norde, E., Eulerian Method for Ice Crystal Icing in Turbofan Engines, Ph.D. thesis, University of Twente, Enschede, the Netherlands, 2017.

[18] Villedieu, P., Trontin, P., and Chauvin, R., "Glaciated and Mixed-phase Ice Accretion Modeling using ONERA 2D Icing Suite," AIAA Paper 2014-2199, Jun 2014, doi: 10.2514/6.2014-2199.

[19] Trontin, P., Blanchard, G., and Villedieu, P., "A Comprehensive Numerical Model for Mixed-Phase and Glaciated Icing Conditions," AIAA Paper 2016-3742, Jun 2016, doi: 10.2514/6.2016-3742.

[20] Iuliano, E., Montreuil, E., Norde, E., Van der Weide, E. T. A., and Hoeijmakers, H. W. M., "Modelling of Non-Spherical Particle Evolution for Ice Crystals Simulation with an Eulerian Approach," Society of Automotive Engineers, Paper 2015-01-2138, Jun 2015, doi: 10.4271/2015-01-2138.

[21] Norde, E., Van der Weide, E. T. A., and Hoeijmakers, H. W. M., "Eulerian Method for In-Engine Icing: With Application to Ice Crystal Trajectories and Impact in a Generic Turbofan Compressor," International Council of the Aeronautical Sciences, Paper 2016-0322, Sep 2016.

[22] Norde, E., Van der Weide, E. T. A., and Hoeijmakers, H. W. M., "Eulerian Trajectory Method and Impingement Model for Non-spherical Ice Crystals," International Conference on Multiphase Flow, Paper 2016-0366, May 2016.

[23] Norde, E., Van der Weide, E. T. A., and Hoeijmakers, H. W. M., "Eulerian Method for Ice Crystal Icing with Application to Particle Trajectories and Accretion on a Three-element Airfoil," AIAA Paper 
2017-3758, Jun 2017, doi: 10.2514/6.2017-3758.

[24] Maxey, M. R. and Riley, J. J., "Equation of Motion for a Small Rigid Sphere in a Nonuniform Flow," Physics of Fluids, Vol. 26, No. 4, 1983, pp. 883-889, doi: 10.1063/1.864230.

[25] Haider, A. and Levenspiel, O., "Drag Coefficient and Terminal Velocity of Spherical and Nonspherical Particles," Powder Technology, Vol. 58, No. 1, 1989, pp. 63-70, doi: 10.1016/0032-5910(89)80008-7.

[26] Ganser, G. H., "A Rational Approach to Drag Prediction of Spherical and Nonspherical Particles," Powder Technology, Vol. 77, No. 2, 1993, pp. 143-152, doi: 10.1016/0032-5910(93)80051-B.

[27] Holzer, A. and Sommerfeld, M., "New Simple Correlation Formula for the Drag Coefficient of Non-spherical Particles," Powder Technology, Vol. 184, No. 3, 2008, pp. 361-365, doi: 10.1016/j.powtec.2007.08.021.

[28] Spalding, D. B., "A Standard Formulation of the Steady Convective Mass Transfer Problem," International Journal of Heat and Mass Transfer, Vol. 1, No. 2-3, 1960, pp. 192-207, doi: 10.1016/00179310(60)90022-3.

[29] Hervo, L., Senoner, J. M., Biancherin, A., and Cuenot, B., "Large-Eddy Simulation of Kerosene Spray Ignition in a Simplified Aeronautic Combustor," Flow, Turbulence and Combustion, In press, available online. doi: 10.1007/s10494-018-9924-4.

[30] Mason, B. J., "On the Melting of Hailstones," Quarterly Journal of the Royal Meteorological Society, Vol. 82, No. 352, 1956, pp. 209-216, doi: 10.1002/qj.49708235207.

[31] Schirmer, R., "Die Diffusionszahl von Wasserdampf-Luft-Gemischen und die Verdampfungsgeschwindigkeit," Beiheft VDI-Zeitschrift, Verfahrenstechnik, Vol. 6, 1938, pp. 170-177.

[32] Hauk, T., Bonaccurso, E., Villedieu, P., and Trontin, T., "Theoretical and Experimental Investigation of the Melting Process of Ice Particles," Journal of Thermophysics and Heat Transfer, Vol. 30, No. 4, 2016, pp. 946-954, doi: 10.2514/1.T4886.

[33] Frössling, N., "On the Evaporation of Falling Drops (Über die Verdunstung Fallender Tropfen," Gerlands Beiträge zur Geophysik, Vol. 52, 1938, pp. 170-216.

[34] Hauk, T., Investigation of the Impact and Melting Process of Ice Particles, Ph.D. thesis, Institute for Fluid Mechanics and Aerodynamics, TU Darmstadt, Darmstadt, Germany, 2016.

[35] Guégan, P., Othman, R., Lebreton, D., Pasco, F., Villedieu, P., Meyssonnier, J., and Wintenberger, S., "Critical Impact Velocity for Ice Fragmentation," Proceedings of the Institution of Mechanical Engineers, Part C: Journal of Mechanical Engineering Science, Vol. 226, No. 7, 2012, pp. 1677-1682, doi: $10.1177 / 0954406211426639$.

[36] Higa, M., Arakawa, M., and Maeno, N., "Size Dependence of Restitution Coefficients of Ice in Relation 
to Collision Strength," Icarus, Vol. 133, No. 2, 1998, pp. 310-320, doi: 10.1006/icar.1998.5938.

[37] Render, P. M. and Pan, H., "Experimental Studies into Hail Impact Characteristics," Journal of Propulsion and Power, Vol. 11, No. 6, 1995, pp. 1224-1230, doi: 10.2514/3.23961.

[38] Hauk, T., Bonaccurso, E., Roisman, I. V., and Tropea, C., "Ice Crystal Impact onto a Dry Solid Wall. Particle Fragmentation," Proceedings of the Royal Society A: Mathematical, Physical and Engineering Sciences, Vol. 471, No. 2181, 2015, pp. 2015-0399, doi: 10.1098/rspa.2015.0399.

[39] Vidaurre, G. and Hallett, J., "Particle Impact and Breakup in Aircraft Measurement," Journal of Atmospheric and Oceanic Technology, Vol. 26, No. 5, 2015, pp. 972-983, doi: 10.1175/2008JTECHA1147.1.

[40] Currie, T. C., Struk, P., Tsao, J.-C., Fuleki, D. M., and Knezevici, D. C., "Fundamental Study of Mixed-phase Icing with Application to Ice Crystal Accretion in Aircraft Jet Engines," AIAA Paper 2012-3035, Jun 2012, doi: 10.2514/6.2012-3035.

[41] Struk, P., Currie, T. C., Wright, W. B., Knezevici, D. C., Fuleki, D. M., Broeren, A., Vargas, M., and Tsao, J.-C., "Fundamental Ice Crystal Accretion Physics Studies," Society of Automotive Engineers, Paper 2011-38-0018, Jun 2011, doi: 10.4271/2011-38-0018.

[42] Currie, T. C. and Fuleki, D. M., "Experimental Results for Ice Crystal Icing on Hemispherical and Double Wedge Geometries at Varying Mach Numbers and Wet Bulb Temperatures," AIAA Paper 2016-3740, Jun 2016, doi: 10.2514/6.2016-3740.

[43] Currie, T. C., Fuleki, D. M., and Mahallati, A., "Experimental Studies of Mixed-Phase Sticking Efficiency for Ice Crystal Accretion in Jet Engines," AIAA Paper 2014-3049, Jun 2014, doi: 10.2514/6.20143049.

[44] Mason, J., Strapp, W., and Chow, P., "The ice particle threat to engines in flight," 44th AIAA Aerospace Sciences Meeting and Exhibit, 2006, p. 206.

[45] Al-Khalil, K., Irani, E., and Miller, D., "Mixed-Phase Icing Simulation and Testing at the Cox Icing Wind Tunnel," AIAA Paper 2003-0903, Jan 2003, doi: 10.2514/6.2003-903.

[46] Pan, H. and Render, P. M., "Impact Characteristics of Hailstones Simulating Ingestion by Turbofan Aeroengines," Journal of Propulsion and Power, Vol. 12, No. 3, 1996, pp. 457-462, doi: 10.2514/3.24057.

[47] Slater, S. A. and Young, J. B., "The Calculation of Inertial Particle Transport in Dilute Gas-Particle Flows," International Journal of Multiphase Flow, Vol. 27, No. 1, 2001, pp. 61-87, doi: 10.1016/S03019322(99)00122-6.

[48] Kah, D., Laurent, F., Fréret, L., de Chaisemartin, S., Fox, R. O., Reveillon, J., and Massot, M., "Eulerian Quadrature-Based Moment Models for Dilute Polydisperse Evaporating Sprays," Flow, Turbulence and Combustion, Vol. 85, No. 3, 2010, pp. 649-676, doi: 10.1007/s10494-010-9286-z. 
[49] Tong, X.-L. and Luke, E. A., "Robust and Accurate Eulerian Multiphase Simulations of Icing Collection Efficiency Using Singularity Diffusion Model," Engineering Applications of Computational Fluid Mechanics, Vol. 4, No. 4, 2010, pp. 483-495, doi: 10.1080/19942060.2010.11015335.

[50] Ekici, K. and Hall, K. C., "Nonlinear Analysis of Unsteady Flows in Multistage Turbomachines Using Harmonic Balance," AIAA Journal, Vol. 45, No. 5, 2007, pp. 1047-1057, doi: 10.2514/1.22888.

[51] Gopinath, A. K., Van der Weide, E. T. A., Alonso, J. J., Jameson, A., Ekici, K., and Hall, K. C., "Three-Dimensional Unsteady Multi-stage Turbomachinery Simulations Using the Harmonic Balance Technique," AIAA Paper 2007-0892, Jan 2007, doi: 10.2514/6.2007-892.

[52] Giangaspero, G., Study of High-Order Energy-Stable Discretization Techniques for Compressible Flows, Ph.D. thesis, Engineering Fluid Dynamics Group, University of Twente, Enschede, the Netherlands, 2016, doi: 10.3990/1.9789036541008.

[53] Capecelatro, J. and Desjardins, O., "An Euler-Lagrange Strategy for Simulating Particle-Laden Flows," Journal of Computational Physics, Vol. 238, 2013, pp. 1-31, doi: 10.1016/j.jcp.2012.12.015. 